\title{
Detection of Microsleep Events with a Behind-the-ear Wearable System
}

\author{
Nhat Pham, Tuan Dinh, Taeho Kim, Zohreh Raghebi, Nam Bui, Hoang Truong, Tuan Nguyen, Farnoush \\ Banaei-Kashani, Ann Halbower, Thang Dinh, Phuc Nguyen, and Tam Vu
}

\begin{abstract}
Every year, the U.S. economy loses more than $\$ 411$ billion because of work performance reduction, injuries, and traffic accidents caused by microsleep. To mitigate microsleep's consequences, an unobtrusive, reliable, and socially acceptable microsleep detection solution throughout the day, every day is required. Unfortunately, existing solutions do not meet these requirements. In this paper, we propose WAKE, a novel behind-the-ear wearable device for microsleep detection. By monitoring biosignals from the brain, eye movements, facial muscle contractions, and sweat gland activities from behind the user's ears, WAKE can detect microsleep with a high temporal resolution. We introduce a Three-fold Cascaded Amplifying (3CA) technique to tame the motion artifacts and environmental noises for capturing high fidelity signals. Through our prototyping, we show that WAKE can suppress motion and environmental noise in real-time by 9.74-19.47 dB while walking, driving, or staying in different environments, ensuring that the biosignals are captured reliably. We evaluated WAKE using gold-standard devices on 19 sleep-deprived and narcoleptic subjects. The Leave-One-Subject-Out Cross-Validation results show the feasibility of WAKE in microsleep detection on an unseen subject with average precision and recall of $76 \%$ and $85 \%$, respectively.
\end{abstract}

Index Terms-Behind-the-ear sensing, Microsleep detection, Drowsiness monitoring, Wearable devices, Cyber-Physical systems.

\section{INTRODUCTION}

$\mathrm{M}$ ORE than 65 million people in the U.S. suffer from Excessive Daytime Sleepiness (EDS) due to sleep deprivation, obstructive sleep apnea, and narcolepsy [1]. EDS often results in frequent lapses in awareness of the environment (i.e. microsleeps). Healthy people with sleep deprivation usually experiences microsleep [1]. Shift workers, night time security guards, and navy sailors with sleep problems have a 1.6x higher risk of being injured, causing 13\% of all work injuries [2]. Sleepy drivers are at a 3x higher risk of an accident causing one in five fatal car crashes [3]. People with sleep apnea also suffers from microsleep. The microsleep issue due to sleep apnea alone leads to a loss of nearly $\$ 150$ million every year due to daily work performance reduction and vehicle accidents [4]. Additionally, more than half of Narcoleptic people are unemployed because of uncontrol-

- N. Pham, T. Nguyen, and T. Vu are with the Department of Computer Science, University of Oxford, United Kingdom.

E-mail: \{firstname.lastname\}@cs.ox.ac.uk

- T. Dinh is with the Department of Computer Science, University of Wisconsin Madison, USA, 53715.

E-mail: \{tuan.dinh\}@wisc.edu

- Z. Raghebi and F. Banaei-Kashani are with the Department of Computer Science and Engineering, University of Colorado Denver, USA, 80204. E-mail: \{firstname.lastname\}@ucdenver.edu

- T. Kim, N. Bui, and H. Truong are with the Department of Computer Science, University of Colorado Boulder, USA, 80302.

E-mail: \{firstname.lastname\}@colorado.edu

- P. Nguyen are with the Department of Computer Science and Engineering, University of Texas Arlington, USA, 76019.

E-mail:vp.nguyen@uta.edu

- A. Halbower is with the Breathing Institute, Children's Hospital Colorado, USA, 80045.

E-mail:ann.halbower@childrenscolorado.org

- T. Dinh is with the College of Engineering, Virginia Commonwealth University, USA, 23220.

E-mail:tndinh@vcu.edu

Manuscript received $\mathrm{XX} X \mathrm{X}, \mathrm{XXXX}$; revised $\mathrm{XX} X \mathrm{X}, \mathrm{XXXX}$.
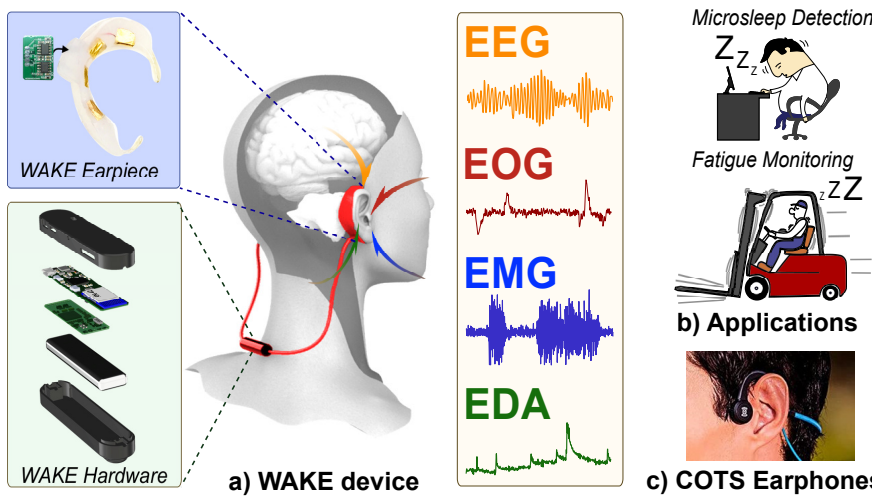

c) COTS Earphones

Fig. 1. Biosignals monitoring from behind the ears concept.

lable microsleep [5]. They often use Amphetamines to keep themselves awake, resulting in many drug overdose cases [6]. Combined, the sleepiness problem of drivers and the workforce costs the U.S. up to $\$ 411$ billion annually [7].

Polysomnography (PSG) and camera-based solutions have been used for microsleep detection. In particular, the Maintenance of Wakefulness Test (MWT) using PSG [8] is the medical gold standard to quantify microsleep based on the electrical signals from the human head, such as brain waves, eyes ball movements, chin muscle tone, and behaviors including eyelid closure, eye blinks, and head nods. This method requires a complicated setup performed by trained technicians in a controlled clinical environment.

Using cameras is another solution to detect microsleep. This approach is the most affordable and common method to detect microsleep for drivers $[9,10]$. The camera-based approach only captures the outer reflection of sleepiness, such as eyelid closure and head nods and ignores the other physiological signatures of sleepiness (e.g., brain and 


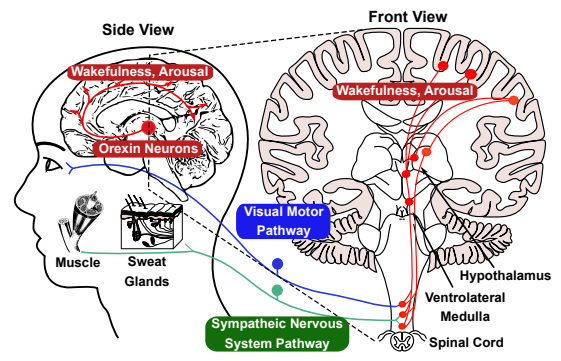

Fig. 2. Wakefulness neural pathway.

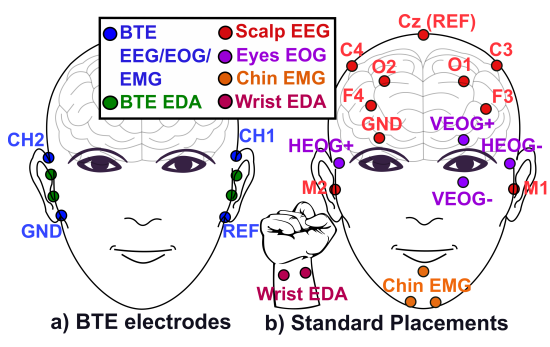

Fig. 3. BTE vs. standard locations.

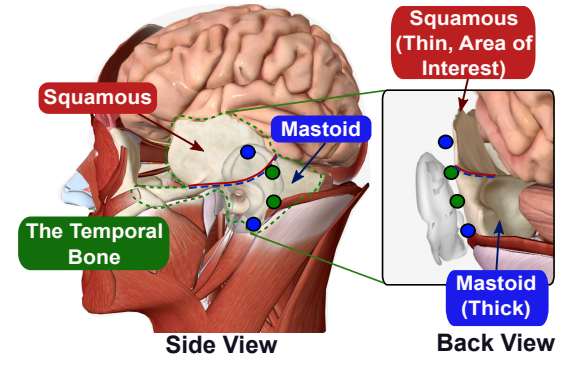

Fig. 4. BTE anatomy. muscle activities) [11]. Thus, it cannot capture microsleep reliably if the episode happens while the subject's eyes still open, which often occurs [12]. Furthermore, using cameras raises strong privacy concerns [13]. Besides, cameras are also limited by environmental light conditions. While wearable cameras can address this issue, wearing a camera on the face is not socially acceptable in daily use. For example, the mixed criticisms of Google Glass on its privacy [14] and form-factor [15] have shown that wearable cameras are not easily accepted by the public.

In this paper, we explore the challenges of building a novel wearable physiological sensing device, called WAKE, for microsleep detection situated only behind the ears (BTE), as illustrated in Fig. 1a. WAKE captures the core biomarkers that are directly related to microsleep from the human head, namely brain waves (EEG), eye movements (EOG), facial muscle contraction (EMG), and skin conductivity (EDA), while being light-weighted and socially-acceptable. While WAKE is currently a standalone device, it could be integrated with earphones and headsets (Fig. 1b), which are already worn daily for listening to music or communication. WAKE includes (1) a wearable design with customized flexible silicon BTE earpieces, electrodes, and a device to sense head-based biosignals, (2) a sensing hardware and software platform to capture different signal types with high fidelity while being robust to motion and environmental noise, and (3) a classification model to detect microsleep.

Challenges. To realize WAKE, we face the following key challenges: (1) heavy noise created by motion and coupled from the environment in daily use is the long-standing challenge limiting the practical uses of wearable biosignal sensing systems, as it is difficult to ensure high fidelity signals; (2) making a wearable, and socially-acceptable device that can capture microsleep is non-trivial because multiple sensors are usually needed to capture its core biomarkers; (3) microsleep detection from behind the ears is an unexplored topic where existing techniques cannot be applied directly; and (4) the BTE biosignals are weak and overlap with each other in the three-orders magnitude range.

Contributions: To overcome the aforementioned challenges, we make the following contributions:

1) We devise a Three-fold Cascaded Amplifying (3CA) hardware technique to make it more practical by ensuring high fidelity signals while mitigating motion and environmental noises.

2) We identify and localize the minimum number of areas behind human ears where biomarkers from the brain, the eyes, facial muscles, and sweat glands can be captured reliably for microsleep detection.

3) We design and prototype a wearable, compact, and so- cially acceptable device that can capture multiple headbased physiological signals.

4) Using a wide range of microsleep biomarkers as features, we developed a hybrid model of a hierarchical classification model and EMG-event-based heuristic rule to detect users' microsleep.

5) We evaluate the proposed system using our custombuilt prototype on 19 subjects. In Leave-One-SubjectOut Cross Validation (LOSOCV), the system obtains $76 \%$ precision and $85 \%$ recall, showing the feasibility for microsleep detection of WAKE on an unseen subject.

Potential Applications: WAKE aims to support a wide range of applications where microsleep detection is essential to ensure user's safety, such as patients with narcolepsy and sleep disorders, heavy machine workers, shift workers, night time security guards, drivers, pilots, and sailors (Fig. 1b). WAKE can also be used for continuous monitoring applications, such as epileptic seizure warning, focus supervising, ADHD monitoring, etc.

\section{Understanding Microsleep}

In this section, we discuss the background knowledge on the physiology and manifestation of microsleep and explain why building a wearable and socially acceptable solution for microsleep detection is challenging.

The manifestation of microsleep. The Orexin system is a wakefulness network throughout the whole central nervous system, as illustrated in Fig. 2. It promotes neuron activity in the mid-brain, the cerebrum, and the visual cortex. These neuronal activities are represented through brain waves, such as fast Beta $(\beta)$ and Alpha $(\alpha)$ waves while the brain is wakeful and conscious, and the slow Theta $(\theta)$ waves when the brain experiences sleepiness. Furthermore, studies on animals [16] have shown that Orexin neurons modulate pupil size, eyelid position, and possibly convergence and eye alignment via motoneurons of multiple muscle fibers. As a result, the wakefulness state is also represented by the movements and activities of the eyes. Additionally, several studies [17] have shown that Orexin regulates wakefulness in the autonomic nervous system (ANS) by activating the ANS through projections to the ventrolateral medulla (VLM) and spinal cord causing the inhibition of sleep. The changes in sympathetic tone are, in turn, represented by changes in facial muscles and sweat gland activity.

Microsleep detection. Microsleep is the temporary episode of losing consciousness and is the key to capturing the transition from wakefulness to sleep. A microsleep episode can last from a few to 30 seconds and people can still wake up after an episode. Microsleep manifests itself both behaviorally (slow-rolling eyes, gradual eye-lid 


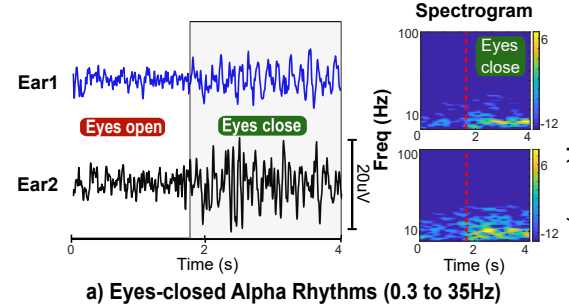

a) Eyes-closed Alpha Rhythms (0.3 to $35 \mathrm{~Hz})$

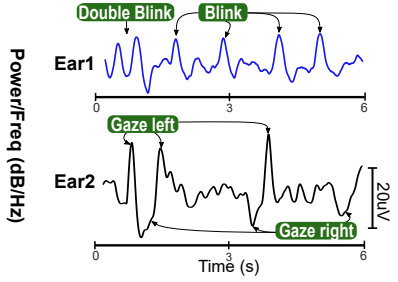

b) Vertical and Horizontal EOG $(0.3-10 \mathrm{~Hz})$

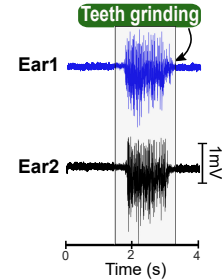

c) Facial EMG $(0.3-100 \mathrm{~Hz})$

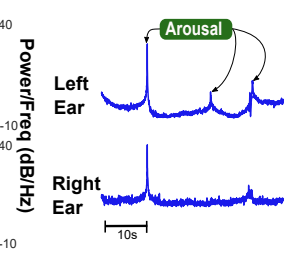

d) $\operatorname{EDA}(0.1-1.5 \mathrm{~Hz})$

Fig. 5. EEG, EOG, EMG and EDA signals captured from behind the ears.

closure, head nods [18]) and electrically (shift in electroencephalography (EEG) from fast $\alpha$ and $\beta$ waves to slower $\theta$ activities [19]). These manifestations link to the inhibition of the Orexin system. Microsleep is extremely dangerous for tasks requiring constant awareness since people who experience MS are usually unaware of them and still believe that they are awake the whole time [20]. This often happens with people who have EDS.

Conventionally, the need for placing multiple sensors on the user's head to capture different biomarkers for accurate microsleep detection makes it challenging to build a wearable and socially acceptable system. As illustrated in Fig. 3b, several electrodes (e.g. at least 9 in the standard PSG system [21]) are usually needed to be placed on the user's scalp to capture brain waves. A wearable camera or 2-4 biopotential electrodes can be placed on the user's eyes to capture eye movements. To capture facial muscle contractions, electrodes are placed on the user's chin. Lastly, sweat gland activity is often captured by electrodes on the wrist or the fingers. With this amount of sensors at different locations on the user's head and face, achieving wearability and social acceptability for microsleep detection is not a trivial task. These studies confirm that there are four key bio-markers that we need to capture for microsleep detection. The remaining questions are (1) where to place the sensors, (2) how many sensors are sufficient, and (3) how the sensors can be made to capture this information? (Section. 3).

Impact of environmental noises and motion artifacts. Various noises and artifacts affect a wearable biosignal sensing system. Motion artifacts and electromagnetic interference from the environment are two major roadblocks for the practicality of the system. Several approaches have been proposed to address the issues of artifacts and noise such as blind source separation with independent component analysis (ICA) or incorporating additional sensors such as inertial measurement units. These approaches, however, depend on a large number of electrodes to provide spatial information, require significant computation, and are difficult to implement in a real-time system [22]. Throughout our in-lab experiments using a PSG device, we found that environmental noises generate significant impacts on the original signal while human motion artifacts completely distort the whole signal, making it not even usable. This requires a novel solution to remove these noises from the signals captured from wearable devices. (Section. 5, 6)

\section{EXPLORING MicrosleEP BIOMARKERS FROM BEHIND THE EARS}

As mentioned in the previous Section, the ear is the intersection of multiple microsleep biomarker sources (e.g. the brain, the eyes, facial muscles, and sweat glands) and is also a natural harbor point where a wearable device could be worn. While recent works on ear-based biosensing have shown the feasibility of capturing individual biosignals (e.g., EEG [23], EOG [24], EEG/EMG [25], and EDA [26]) from the area around and behind the ears, monitoring microsleep-related biosignals with a wearable form-factor has not been explored before. Thus, it is unclear about (1) where are the best places for EEG, EOG, EMG and EDA sensors to achieve both wearability and sensing sensitivity, (2) what is the minimum number of required electrodes, and (3) what are the unique characteristics of BTE signals?

The BTE electrodes placements. From our study on the ear anatomy, we derive the best sensor placement locations for microsleep detection, as shown in Fig. 3a and Fig. 4. At these locations, we can capture signals coming from the mid-brain area (EEG), eye movements (EOG), facial muscle contractions (EMG), and sweat gland activities (EDA). These sensor locations allow us to design a socially-acceptable wearable device that is well-hidden behind the user's ears just like commercial off-the-shelf (COTS) earphones.

Fig. 4 illustrates the anatomy of the temporal bone covering the whole BTE area. It consists of two major parts, i.e. the Squamous and Mastoid processes. To capture EEG generated by the mid-brain area, we would want to place the electrodes on the Squamous process, which is the thin upper part of the temporal bone. This makes electrodes as close to the brain as possible. Two electrodes, i.e. channel 1 on the left ear and channel 2 on the right ear, are used to capture EEG on both sides of the brain. To capture EOG, i.e. vertical EOG (vEOG) and horizontal EOG (hEOG), we need to maximize the vertical and horizontal distance between each pair of electrodes, respectively. Thus, we place the reference electrode on the Mastoid process, which is the thick lower part. With this setup, channel 1 can pick up eye blinks and up/down movements, while channel 2 can capture the eyes' left and right movements. Additionally, both channel 1 and 2 can capture most of the facial muscle activities that link to the muscle group beneath the area behind the ears. Since EEG, EOG, and EMG are biopotential signals, we can use the same electrodes. Thus, we only need four electrodes, including two signal electrodes, a reference, and a common ground, to capture them. Capturing EDA behind the ear is promising because it has high sweat gland density [27]. As sweat gland activities are not symmetric between two halves of the body, placing two electrodes on each ear is necessary to reliably capture EDA.

Examining BTE signals. Signals captured from BTE electrodes resemble the most important biomarkers of microsleep that we would expect from standard electrodes placements (i.e, EEG, EOG, EMG, and EDA), as shown in Fig. 5. In particular, Fig. 5a presents the $\alpha$ rhythms seen 


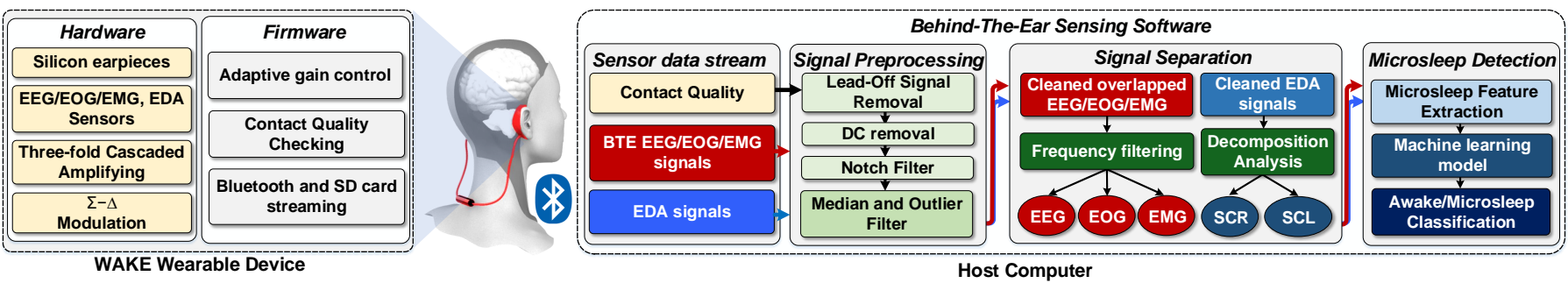

Fig. 6. WAKE system overview.

on both BTE leads when the eyes are closed. Similarly, the same features, such as eye blinks, left gaze, right gaze, teeth grinding, and emotional arousal can be captured with BTE electrodes, as shown in Fig. 5b, c, and d, respectively. Thus, the results confirm that we could capture the aforementioned microsleep features from only behind the ears.

There are unique challenges to BTE signals. First, BTE signals are much smaller than the ones expected with standard placements. Particularly, the amplitude of EEG and EOG captured from BTE are all less than $50 \mathrm{uV}$, which are much smaller than standard placements $(100-500 \mathrm{uV})[28]$. This is probably because BTE electrodes are far from the signal sources of EEG and EOG. Secondly, we notice a significant amplitude difference (i.e. three orders of magnitude) between BTE EEG/EOG and EMG signals, as BTE EMG events could be as strong as a few millivolts. Moreover, the spectrogram in Fig. 5 shows that BTE EMG events have very strong power in all frequency bands from 0.3 to $100 \mathrm{~Hz}$. As we use the same BTE electrode to capture EEG, EOG, and EMG, addressing the overlap of the three signals is not trivial. Low amplitude BTE EEG/EOG signal overlapped with EMG making it challenging to ensure high fidelity microsleep features while being robust against environmental noise and motion artifacts.

\section{System OVerview}

We design WAKE to include four main components (Fig. 6): (1) a motion mitigation sensing hardware using the 3-folds Cascaded Amplifying (3CA) technique, (2) a firmware adaptively amplifies of the signals; (3) a software running on a host device to process data from BTE sensors and detect user's microsleep; and (4) an ear-worn device designed for long-time usage.

WAKE hardware. We design a highly sensitive sensing circuit (Fig. 7) to capture the brain waves (EEG), polarization signal created by eyeball activities (EOG), facial muscle contractions (EMG), and electrodermal activities (EDA). In WAKE, we derive an approach called 3CA, allowing the system to minimize the impact of motion artifacts and environmental noises in real-time at hardware and firmware levels. The key idea is to utilize multiple buffering and amplifying stages with precision buffers and instrumentation amplifiers to address the effects of electrode fluctuation, cable shaking, and environmental interference (Sec. 5).

WAKE firmware. WAKE firmware is designed to control our sensing hardware so that data from four main sensors: EEG, EOG, EMG, and EDA can be captured reliably (Sec. 6). The key challenges are that the signals are often weak and overlap each other. Thus, we design the firmware with three main components (1) adaptive gain control (AGC), (2) contact quality checking, and (3) Bluetooth and SD card streaming. AGC addresses the overlapping issue by dynamically changing the amplifier gain based on different signal types. Electrode contact quality is monitored so that we can detect and remove noisy signals created by loose electrodes. The collected data is streamed over Bluetooth and to an SD card for later analysis.

WAKE algorithms. WAKE algorithms are implemented on a host device (i.e., mobile phones, laptops, etc.). Upon receiving the signals from the WAKE ear-worn device, the data are separated into different streams and ready for further processing. There are three main data streams are collected including a BTE EEG/EOG/EMG signals, EDA signal, and contact impedance signal. During signal preprocessing, the DC, electricity noises, and other noises are removed by DC removal, notch, median, and outlier filters, respectively. The clean EEG, EOG, EMG, and EDA signals obtained from pre-processing are then used for microsleep classification. The features extracted from these signals are later used to together with a set of machine learning algorithms to detect microsleep.

WAKE earpieces. WAKE system is designed for comfortable, reliable, cost-effective, and continuously collecting behind the ear signals. To realizing that goal, we design the earpieces by carefully sketching the device architecture and then implementing them using off-the-shelf components. The earpieces materials were also carefully selected ensuring good contacts between the electrodes and the human skin as well as allowing it to be comfortably worn by users. We also validated and identified the most proper electrode materials that provide the highest sensitivity (Sec. 8).

In the next section, we will discuss our proposed solution to address one of the most important challenges of designing a reliable wearable device: "how to cancel the noises created by human motion artifacts and coupled from the environment?"

\section{Mitigating Impact of Motions \& Noise - A HARDWARE SOLUTION}

Noises created by motion and coupled from the environment are important challenges that we need to overcome to ensure the reliability and practicality of WAKE. These noise span across all frequency of interest and are highly unpredictable, making their removal non-trivial from the signal by software methods such as filtering or ICA. In literature, Active Electrodes (AE) [29] have been proposed to mitigate motion artifacts and environmental interference. However, conventional $\mathrm{AE}$ does not consider the unique characteristic of BTE signals, which are (1) weak EEG and EOG signal amplitudes, (2) signals overlap with three orders of magnitudes difference, and (3) limited spaces for BTE electrodes. We propose a technique called Three-fold Cascaded Amplifying (3CA) on the electrical pathway of 


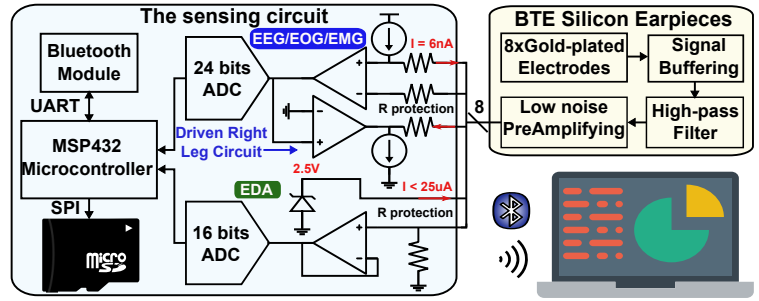

Fig. 7. WAKE hardware module.

WAKE. Fig. 8 presented the model for the 3CA technique with three stages: (1) Stage 1 - Unity Gain Amplifying, (2) Stage 2 - Feed Forward Differential PreAmplifying (F2DP), and (3) Stage 3 - Adaptive Amplifying. The 1st and 2nd stages are implemented on our BTE earpieces, while the 3rd stage is implemented on the sensing circuit and its firmware.

Stage 1 - Unity-gain amplifying. The root cause of motion artifacts lies in the fluctuations of the wires and micromovements of electrodes [30]. These fluctuations create changes in the electrical pathway resulting in measurement noise. We address the motion artifacts by introducing the first stage: unity-gain amplifying (a.k.a buffering). Considering the reference circuit model, as in Fig. 8, $V_{s}$ is the signal source from the ears, $C w$ is inherent capacitance on signal cables, and $Z_{c}$ is the skin-electrode contact impedance. $V_{o}$, $A, Z_{i}, R_{i}, C_{i}, Z_{o}, C_{p}$ are output voltage; ideal voltage gain; input impedance, resistance, and capacitance; output impedance; and parasitic capacitance of each amplifier.

Since the biosignals are extremely weak (i.e., $\mu \mathrm{V}$ level), instrumentation amplifiers are usually used to amplify the signals, making them available for further processing. When an instrumentation amplifier is used, we can model the effect of motion artifacts by using the voltage gain rule ( $V_{o}=A * V_{i}$ ) and Kirchhoff's current and voltage laws (1) at the input of the amplifier:

$$
\left(V_{s}-V_{i}\right) /\left(Z_{c 1}+Z_{c 2}\right)-\left(V_{i}\right) /\left(Z_{i}\right)+j \omega C_{p}\left(V_{o}-V_{i}\right)=0 .
$$

By eliminating $V_{i}$ from Eq. 1, we have a relationship among the actual gain $\left(G=V_{o} / V_{s}\right)$ of the circuit, skin-electrode contact impedance $\left(Z_{c 1}, Z_{c 2}\right)$, and the inherent capacitance on signal wires $\left(C_{w}\right)$ :

$$
G=\frac{A}{1+\left(Z_{c 1}+Z_{c 2}\right)\left(\frac{1}{R_{i}}+j \omega\left(C_{w}+C_{i}-(A-1) C_{p}\right)\right)} .
$$

As motions happen, cable sway and electrode movement create the fluctuation on $C_{w}$ and $Z_{c 1}+Z_{c 2}$, respectively. This results in the fluctuation of the actual gain $(G)$. To minimize the fluctuation effect of $C_{w}$ (generated by triboelectric processes and change of parasitic capacitance in the measurement network [31]), we can use an op-amp buffer for each electrode to convert the high impedance lines $\left(Z_{c 1}, Z_{c 2}\right)$ to approximately zero $\left(Z_{o 1}, Z_{o 2} \approx 0\right)$. Rewrite Eq. 2 for the op-amp buffer in the first stage, we have

$$
G=\frac{A_{1}}{1+Z_{c 1}\left(\frac{1}{R_{i 1}}+j \omega\left(C_{w 1}+C_{i 1}-\left(A_{1}-1\right) C_{p 1}\right)\right)}=\frac{A_{1}}{1+Z_{c 1} \gamma}
$$

Ideally, the effect of $Z_{c 1}$ fluctuation can be removed if we can satisfy the following equation: $\gamma=0$. While it is very challenging to achieve in practice, we still can make $\gamma$ as close to 0 as possible. This could be done by using an ultrahigh input impedance buffer, where $A_{1}=1, R_{i 1} \rightarrow \infty$,

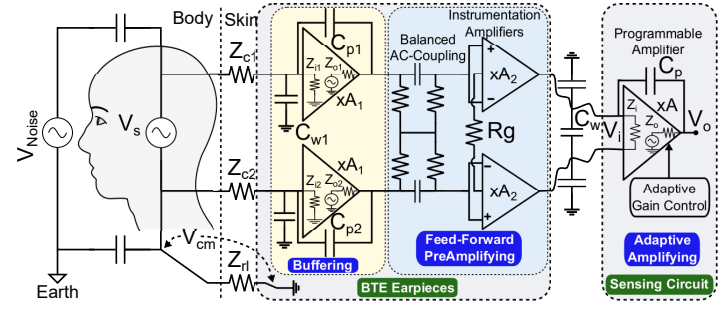

Fig. 8. 3CA model.

and $C_{i 1} \rightarrow 0$ in our first stage. Putting a buffer circuit directly on the electrodes is the best way to minimize $C_{w 1}$. However, this is not desirable, as we have limited space for our BTE electrodes. We notice that as long as we keep $C_{w 1}$ small and stable, putting the circuit directly on the electrode is possible. This is done by fixing the connection between each electrode and its buffer in a stable structure to avoid triboelectric noise and shielding it by using a micro-coax shielded cable. By driving the shield with the same voltage as the signal from the output of the amplifier, we effectively minimize $C_{w 1}$. Up to this point, the unity-gain amplifying stage can remove the impact of human motion artifacts.

The use of ultra-high input impedance buffers at this stage also brings another benefit, i.e., reducing environmental noise coupled into the measurement because of the imbalance among signal lines. When a common-mode voltage $\left(V_{c m}\right)$ is introduced to the subject body by an environmental noise source $\left(V_{\text {noise }}\right)$ (Fig. 8$)$, a portion of $V_{c m}$ leaks into our differential measurement and becomes noise $\left(V_{n}\right)$ because of impedance mismatch, i.e. $V_{n}=$ $V_{c m}\left(\overline{Z_{c}} / \overline{Z_{i}}\right)\left(\left(\Delta Z_{c} / \overline{Z_{c}}\right)+\left(\Delta Z_{i} / \overline{Z_{i}}\right)\right)$ [32]. During a longterm measurement in practice, contact impedance can easily deteriorate (e.g., from $<10 \mathrm{k} \Omega$ to several hundreds of $\mathrm{k} \Omega$ ) because of drying gel or unstable contacts [33]. Junctiongate field-effect transistor (JFET) input amplifier is a good solution to address this issue thanks to its extremely high input impedance $\left(10^{12}-10^{15} \Omega\right)$. Thus, we can significantly reduce $V_{n}$ making our measurement more robust against impedance mismatch. The signals, however, need to go through another stage to remove all the environmental noise, as described in the following discussion.

Stage 2 - Feed Forward Differential PreAmplifying (F2DP). To ensure robustness against environmental interference, intuitively, we would want to preamplify our weak and overlapped BTE signals before driving the cables to our sensing circuit. Conventionally, if an amplifier with positive gain $(>1)$ is used, the equation $\gamma=0$ cannot be satisfied, making the system prone to motion artifacts. Furthermore, electrode contact impedance mismatch, which is often seen in practice, leads to the a gain mismatch among electrodes, as shown in Eq. 3. Gain mismatches between two electrodes will allow more common-mode noise to be coupled into the system. By dividing into unity-gain and F2DP stages, we overcome this challenge since the input impedance of F2DP is effectively close to 0 . Thus, the effect of contact impedance will not affect the gain in the next stages.

Inspired by the robustness against noises of balanced audio systems where preamplified differential signals are generated before transiting over wires, differential signaling is employed in our design. We apply the Feed-Forward (FF) differential amplifying technique, which has been shown 


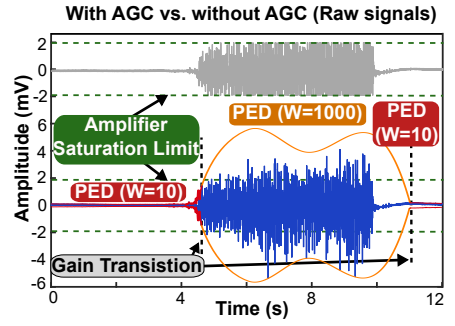

Fig. 9. EMG saturation w/o AGC.

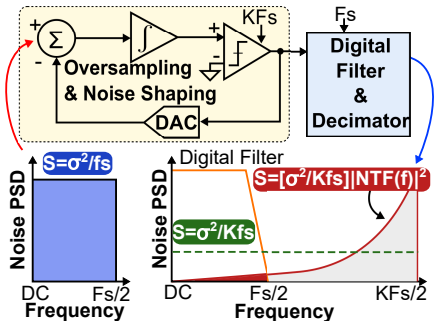

Fig. 10. $\Sigma-\Delta$ modulation.

by simulation in [34] to significantly increase the CommonMode Rejection Ratio (CMRR), i.e. the ability to reject noise coupled from the environment, than using the conventional Driven Right-Leg circuit (DRL) by $49 \mathrm{~dB}$. The FF topology used in [34], however, is not practical because its stability suffers from gains mismatch when two different gain resistors are used in the proposed topology. Mismatch in these resistors causes the output common-mode level to move with the output signal, resulting in distortion [35]. Thus, we employ the cross-connection technique where only one gain resistor (i.e., $R_{g}$ in Fig. 8 ) is needed to set the gain for two FF instrumentation amplifiers in our F2DP. After F2DP, fully differential and preamplified signals are produced making them robust against environmental interference while driving the cables to the sensing circuit.

F2DP only works when the DC component is removed completely. We found that the traditional high pass RC filter approach is not efficient in removing the DC component (100x larger than signals of interest) because it introduces an additional ground-path and component mismatch, which reduces the efficiency of rejecting environmental noise of F2DP. Balanced AC-coupling topology [36] is a best-fit solution to overcome these challenges because it does not introduce any additional ground-path and minimizes the component mismatch since the pole and zero of the filter cancel themselves out. In particular, considering 'Balanced AC-coupling' components in Fig. 8, this topology does not include the ground-path, thereby eliminating its side-effects. Moreover, RC components are never precise in practice (approximately from $1 \%$ to $20 \%$ error for a capacitor) and their mismatch problem is difficult to solve. The chosen balanced AC-coupling topology dampens these mismatches by canceling the redundant poles and zeros created by component mismatch [36].

Stage 3 - Adaptive Amplifying. After the previous stage, the system can reliably collect BTE signals; we are now solving the problem of our BTE signals themselves. The unique challenge that we need to address with our BTE signals is the significant amplitude range differences between EEG/EOG and EMG signals (i.e. from $\mathrm{uV}$ level to $\mathrm{mV}$ level for EEG/EOG and EMG signals, respectively). This challenge has not been considered in the traditional EEG system, as EEG electrodes are placed far away from EMG sources. The difference leads to signal saturation at the ADC on the sensing circuit when EMG signals are amplified with the same gain as EEG/EOG signals. The CMRR of the amplifier is presented by the following equation: $C M R R=10 * \log \frac{A_{d}^{2}}{A_{c m}^{2}}$, where $A_{d}$ and $A_{c m}$ are the differential and common-mode gain, respectively. In an instrumentation amplifier, $A_{c m}$ is a constant depending on the internal resistors. Thus, CMRR only depends on $A_{d}$. Since the difference between EMG and EEG/EOG could be as large as three orders of magnitude, setting the gain too low to avoid EMG saturation will also significantly lower CMRR (up to $60 \mathrm{~dB}$ ), increasing the noise floor to a level where EEG/EOG signal cannot be captured. We found that the gain needs to be dynamically adjusted in real-time so that both small EEG/EOG and large EMG signals are captured with high resolution.

\section{Signal Processing}

\subsection{WAKE On-board processing.}

Adaptive Gain Control. As aforementioned in Sec. 5, one important and unique challenge in ensuring high fidelity BTE signals is the large difference in the amplitude range (which could be up to three orders of magnitude) between EEG/EOG and EMG signals. Thus, the analog gain of our sensing circuit needs to adapt dynamically with the changes in signal amplitude. Fortunately, we observe that (1) EMG events do not happen frequently, (2) EMG events can happen quickly with strong amplitude changes, and, (3) signal amplitude during an EMG event is stochastic and can vary significantly. Understand these characteristics, we then design our AGC to (1) keep the gain at maximum for EEG/EOG signals while there is no significant EMG events, (2) react quickly to the abrupt increase of amplitude to detect EMG events but (3) react slowly to the decrease of amplitude while an EMG event is still happening to avoid gain oscillation. Peak Envelope Detector (PED) and Square Law Detector (SLD) are two popular AGC techniques [37] that fit with our needs. We use PED because of its low computational complexity. If there is no EMG event, we use a small window size so that PED can react quickly while a larger window size is used during an EMG event to avoid gain oscillation.

We can choose the PED window size based on the property of EMG signals, sampling rate, desired response time, and the device's computational resource. According to $[38,39]$, EMG signal has the frequency range of $1-500 \mathrm{~Hz}$ and is most dominant in between $50-150 \mathrm{~Hz}$. Thus, with the sampling rate of $1000 \mathrm{~Hz}$, we can cover the EMG signal range by using the small and large window sizes of 2 and 1000 samples, respectively. With the large window size of 1000 samples, our AGC can guarantee that the gain will be adjusted within one second after the EMG event has ended. This response time is acceptable in our application as a microsleep can last at least a few seconds. The small window size of two samples can make our AGC very sensitive even with the fastest EMG signal, but it also increases our processor load. Since the dominant EMG signal power is in the range of $50-150 \mathrm{~Hz}$, we can increase the small window size to reduce the load. During a gain transition, the amplifier needs to be stabilized before new data can be obtained. We can interpolate missing samples with a lightweight linear interpolation. Fig. 9 shows an EMG event is captured without saturation by using AGC with the small and large window size of 10 and 1000 samples, respectively.

$\Sigma-\Delta$ modulation. To ensure high signal quality during the quantization process, we employ the $\Sigma-\Delta$ modulation, which could be found in high precision Analog-to-Digital Converters (ADCs). Low quantization noise is achieved by utilizing oversampling, noise shaping, digital filtering, and 


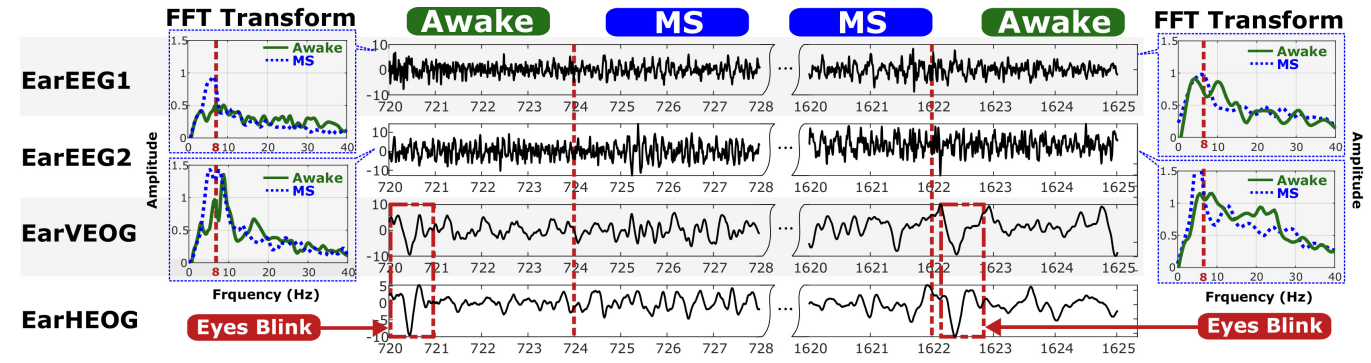

Fig. 11. Signals captured by WAKE during the transition between awake and microsleep.

decimation, as illustrated in Fig. 10. With a given ADC having the resolution of $b$ bits, the full signal scale FS, and the quantization error $(\varepsilon)$ is assumed to be a stationary, random process; the quantization noise is a constant $\left(\sigma_{\varepsilon}{ }^{2}=F S^{2} /\left(3 * 2^{2 b}\right)\right)$ [40]. Without a noise shaping function, the quantization noise spreads out uniformly over the Nyquist spectrum. Thus, the power spectral density is also constant, i.e. $S_{e}(f)=\sigma_{e}^{2} / f_{s}$ with $f_{s}$ is the sampling frequency. Oversampling (by a factor of K) widens the Nyquist spectrum, thereby, reducing the quantization noise energy in the spectrum of interest, i.e., $S_{e}(f)=\sigma_{e}{ }^{2} /\left(K f_{s}\right)$. To further reduce the noise inside the spectrum of interest, we pass the signal through a noise shaping function called the $\Sigma-\Delta$ modulator. Fig. 10 presents a first-order $\Sigma-\Delta$ modulator constructed as a negative feedback loop. By using Ztransform, we have the noise transfer function (NTF) of the loop to be $\operatorname{NTF}(z)=1-z^{-1}$ [40]. Converting the NTF to the frequency domain by using trigonometric identities, we have $N T F^{2}(f)=4 \sin ^{2}(\pi f / f s)$. Thus, the new noise power spectral density is $S_{e}(f)=\sigma_{e}{ }^{2} /\left(K f_{s}\right) *|N T F(f)|^{2}$. Similarly, we can generalize the NTF equation for a Nthorder $\Sigma-\Delta$ modulator as $N T F^{2}(f)=[2 \sin (\pi f / f s)]^{2 N}$. As illustrated in Fig. 10, a $\Sigma-\Delta$ modulator can shift the noise energy to the high frequency of the spectrum. A digital filter removes the noise power outside our spectrum of interest. The signal is then decimated to the required sampling rate before outputting the results. Thus, we can achieve a low noise floor for our measurement.

Contact quality checking. To monitor contact quality, we inject a small sinusoidal AC current (i.e. $I=6 n A$, $30 \mathrm{~Hz}$ ) through the skin-electrode contact. By measuring the response voltage, we can calculate the contact impedance by this equation: $Z_{30 \mathrm{~Hz}}=V_{R M S, 30 \mathrm{~Hz}} / I-R_{\text {protection }}$. We follow the clinical standards as in [21] stating the acceptable upper limit of electrode impedance is $10 k \Omega$ to achieve optimal biosignals recording. This can be achieved with standard procedures of skin preparation and conductive gel application. As the electrode impedance can varies over time because the electrode contact loosens or the gel dries out, we can notify the user to adjust contact or reapply the gel if the impedance is higher than our defined threshold.

\subsection{WAKE Physiological Signals Extraction}

In WAKE, each sensor data (EEG/EOG/EMG, and EDA) is pre-processed at the host device corresponding to their own characteristics before putting it into the signal analyzing procedure. We show the examples of changes in those signals between microsleep and awake states in Fig. 11. We apply to all sensor data the notch filter to remove $50 / 60 \mathrm{~Hz}$ power line interference, linear trend removal to avoid DC drift, and outlier filters to remove spikes and ripples.
Collecting EEG/EOG/EMG signals. WAKE's mixedbiosignals include EEG, EOG, and EMG, which are in the frequency range of $4-35 \mathrm{~Hz}, 0.1-10 \mathrm{~Hz}$, and 10-100 $\mathrm{Hz}$, respectively. We apply different bandpass filters to split the mixed BTE biosignals into the signals at the frequency range of interest. In particular, we extract wakefulness-related EEG bands (i.e $\theta, \alpha$, and $\beta$ waves) using $4-8 \mathrm{~Hz}, 8-12 \mathrm{~Hz}, 12-35$ $\mathrm{Hz}$ bandpass filters, respectively. We extract horizontal EOG (hEOG) for eye movement and vertical EOG (vEOG) for eye blink using $0.3-10 \mathrm{~Hz}$ filters. A $10-100 \mathrm{~Hz}$ bandpass filter and a median filter are then applied to the mixed signals to extract the EMG band and get rid of spikes and other excessive components.

Collecting EDA signal. EDA signal is the superposition of two different components, skin conductance response (SCR) and skin conductance level (SCL) at the frequency range of $0.05-1.5 \mathrm{~Hz}$ and $0-0.05 \mathrm{~Hz}$, respectively. Even though EDA signals have fast responses, they are very slow to decline to baseline. Thus, if another response happens right after the first response, the signal level will increase even more. Thus, frequency filtering is not effective to separate EDA signal. To address this, we employ an nonnegative deconvolution technique proposed in [41] to decompose EDA into SCR and SCL components.

\section{Algorithms}

We present two classification methods: (1) feature engineering-based classification, and (2) deep learning on raw data. Feature-based classification is built on wellstudied microsleep features with off-the-shell machine learning models. While this approach may help the learning procedure more stable, interpretable with less amount of data, it is labor-intensive for processing features. The second approach using deep learning tackles this issue, and has been shown to achieve the state-of-the-art performance on micro-sleep detection.

\subsection{Classification based on Feature Engineering}

EMG active events detection: Microsleep appears when the body is relaxed. A strong EMG signal can have significant power across all frequency bands of interest (discussed in Sec. 3). It will contaminate our BTE EEG and EOG signals rendering them unusable. We detect the active event based on the sum of all frequency bands in the spectrogram. For each data signal, we use the first 10 seconds as the groundbased noise. Any data whose total spectrum energy is $10 \%$ larger than that of ground-based is an active event.

Feature Extraction: We divide the collected time series data from each source into fixed-size epochs. The selected features are extracted from each epoch for classification. 


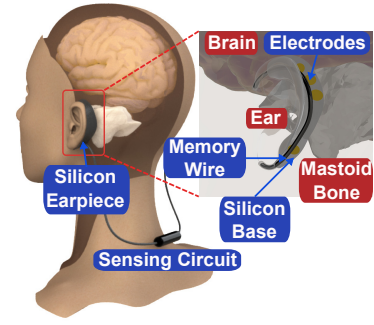

Fig. 12. 3D model.

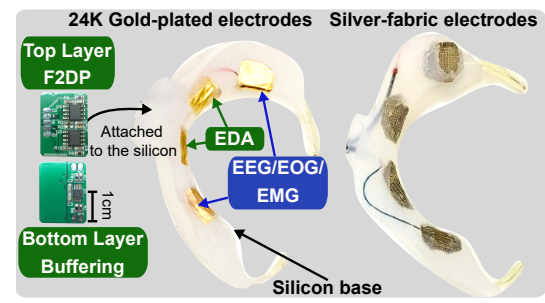

Fig. 13. BTE silicon earpieces.

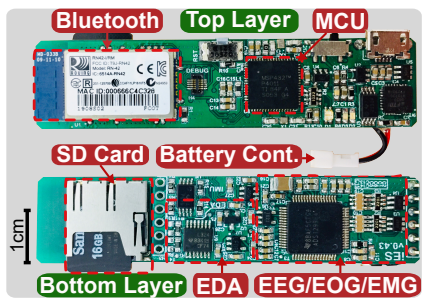

Fig. 14. WAKE circuit.

Temporal features: This category includes typical features used in the literature for time series data analysis in the temporal domain, namely mean, variance, min, max, hjorth, skewness, and kurtosis. In microsleep detection, EOG, EMG, and EDA signals are often analyzed in the time domain due to their considerable variation in amplitude and lack of distinctive frequency patterns [42]. Those six temporal features are extracted from each of hEOG, vEOG, EMG, and EDA signals for a total of 24 temporal features. We use wavelet decomposition for the hEOG signal to extract saccade features, namely mean/max velocity, mean/max acceleration, and amplitude range. Eyeblink features, namely mean/max amplitude, peak closing velocity, peak opening velocity, and closing time are extracted from the vEOG signal.

Spectral features: The spectral features are extracted to analyze the characteristics of the EEG signal because brainwaves are generally available in discrete frequency ranges at different stages. Those features include the ratio of powers, absolute powers, $\theta / \beta, \alpha / \beta, \theta / \alpha$, and $\theta /(\beta+\alpha)$. Accordingly, 14 features are extracted from each channel of EEG providing 28 spectral features in total.

Non-linear features: Bioelectrical signals show various complex behaviors with nonlinear properties. In particular, the chaotic parameters of EEG can be used for microsleep detection. The discriminant ability of nonlinear analyses of EEG dynamics is demonstrated through the measures of complexity such as correlation dimension, Lyapunov exponent, entropy, fractal dimension, etc. [43], with the last two features proven to be most informative. In this study, we extract these two non-linear features for each of the two EEG channels (a total of four features).

Feature Selection: When all features are used altogether, irrelevant correlated features or feature redundancy can degrade the performance. Therefore, we adopt three feature selection methods, including Recursive Feature Elimination (RFE), L1-based, and tree-based feature selection to select the set of most relevant features. RFE is a greedy optimization algorithm that removes the features whose deletion will have the least effect on training error. L1-based feature selection is used for linear models, including Logistic Regression and SVM. In our linear models, we use the L1 norm to remove features with zero coefficients. Finally, the feature importance ranking generated by the tree-based model is used to eliminate irrelevant descriptors.

Microsleep Classification: Various classification methods from Support Vector Machine (SVM), Linear Discriminant Analysis (LDA), Logistic Regression (LR), Decision Tree (DT) to ensemble methods like RandomForest or AdaBoost have been proposed in the literature for awake and microsleep classification, each shown to be effective in specific settings [44]. To cope with the high complexity of

our collected signals, we developed a hierarchical stack of three base classifiers. Our hierarchical model consists of a Random Forest classifier (with 50 estimators) in the first layer, Adaboost classifier (with 50 tree estimators) in the second layer, and SVM (with RBF kernel) in the last layer. Specifically, for the first two layers, we only keep the predictions with high probabilities $(>0.7)$ and transfer the rest of samples to the next layer. In the last layer, SVM classifies all of the remaining samples. We also apply a heuristic rule to the final predication based on the knowledge that an EMG event is likely to leads to an 'awake' event. The results of our empirical analysis are presented in Sec. 9, which highlights the overall accuracy of the performance and proves the efficiency of the proposed classification model.

\subsection{Deep Learning on Raw Data}

Deep neural networks (DNNs) is a branch of Artificial Neural Network which can model highly complex nonlinear functions. An advantage of DNNs compared to other machine learning algorithms is its ability to automatically learn features from raw inputs. Also, DNNs usually require less manual adjustments even though they need more data and computations, thus being easier to deploy and maintain.

Modified Sorsnet: while deep learning community has extensively studied perceptual data (e.g., image, audio, text), brain signals are still under-explored. Recent works from sleep staging and microsleep detection have demonstrated the effectiveness of DNNs, especially Convolutional Neural Networks (CNNs) in learning meaningful patterns of EEG signals. We make use of a relevant CNN architecture proposed by [45] as it achieves the state-of-the-art performance for sleep classification using a single EEG channel. This model contains $121-\mathrm{D}$ convolutional blocks followed by 2 fully connected layers. Each block consists of a 1-D convolution layer, a BatchNorm layer and a ReLU activation function. As our data contain 24 1-D signals, we modify this architecture by stacking these signals into a 24-channel signals and update the input channel of the first convolutional layer. For training, we use cross-entropy loss [46], Adam optimizer [47] with $\beta_{1}=0, \beta_{1}=0.99$, and Lambda scheduler for the learning rate decay. We train the SorNet model with 200 epochs. To derive the best hyper-parameter, we apply the grid search for learning rate in $\left[1 e^{-4}, 0.1\right]$, and find out that learning rate 0.002 works well for us in most of the cases. We use an additional validation set on the precision score to overcome the over-fitting and pick the best model.

\section{IMPLEMENTATION}

Earpieces' Material. We design the BTE biosensing earpieces by attaching electrical conductive material on top of a silicone base as illustrated in Fig. 12. The silicone material 


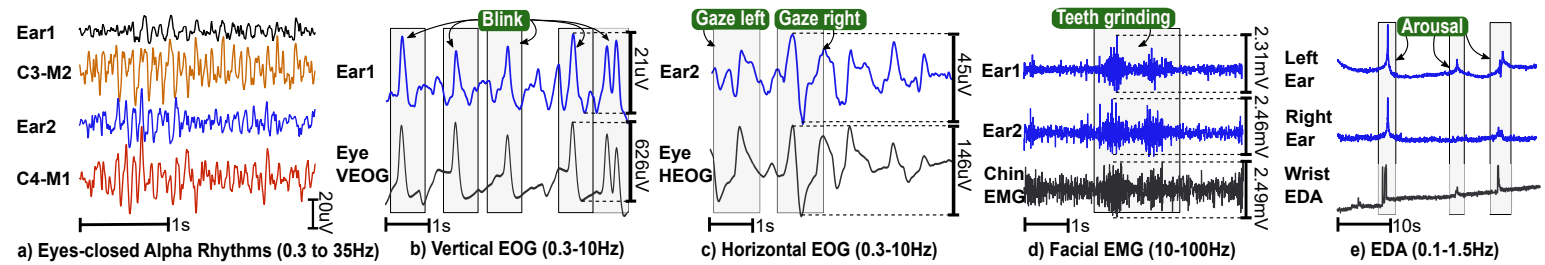

Fig. 15. BTE EEG, EOG, EMG and EDA signals vs. ground-truth.

(Dragon Skin 10) is chosen so that the earpiece can fit with the curve created by the mastoid process, while being comfortable and alterable with different user's ears (Fig. 12, 13). Furthermore, the chosen silicone material is skin safe and does not create irritation to the user's ears. The silicone base is molded based on the average size of the human ear [48]. To maintain a good contact between the electrodes and skin, we put a memory wire inside the silicone base. The memory wire creates a grip on the wearer's ears, pushing the silicone against the skin. It helps the earpieces be usable for different human ear sizes and shapes.

Electrodes' Material. We evaluated three different materials for the electrodes attached to the earpieces including (1) silver fabric, (2) copper pad and (3) gold-plated copper pad. The silver fabric electrodes are highly conductive and can make good contact with the skin thanks to the flexibility of the fabric, but the silver gets tarnished quickly because of the skin oil and sweat. Thus, the contact quality degrades after several uses as the resistance increases dramatically from less than $1 \Omega$ to several hundred $k \Omega$. Similarly, copperbased electrodes also degrade quality after several uses. We address that issue by plating gold liquid over the copper electrodes, because the gold-plated electrodes are more resistant to skin oil and sweat. In addition, gold is well-known to be chemically inert. Thus, the skin allergy with gold is extremely rare. The resistance of the gold-plated electrodes is always less than $1 \Omega$. To enhance contact conductivity and adhesion, we apply Weaver's Ten20 conductive paste on the electrodes before wearing the earpieces. The contact impedance between the electrodes and the skin is also measured to be in the range from 5 to $10 \mathrm{k} \Omega$ at $30 \mathrm{~Hz}$ with a proper skin preparation. This impedance value satisfies the clinical standards [21], which state the acceptable upper limit is $10 \mathrm{k} \Omega$, to achieve optimal recordings.

Putting Things Together. We use the low power, precision AD8244 JFET-input buffer to implement our Stage 1 of 3CA. The AD8244 device has unity gain, very high input resistance (i.e., 20T $\Omega$ ), and very low input capacitance (i.e., $12 \mathrm{pF}$ ) so that the effect of motion and impedance mismatch can be minimized as pointed out in Sec. 5. The precision, instrumentation amplifier AD8222 is used to implement our Stage 2 (F2DP). The preamplifying gain is chosen at 100 so the full range of the ADC (i.e., $-2.5 \mathrm{~V}$ to $2.5 \mathrm{~V}$ ) is utilized. We use an ultra-low noise amplifiers and 24-bits ADC chip ADS1299 to digitize the signals. The ADS1299 provide an integrated second-order $\Sigma-\Delta$ modulator. It samples the input signal at $1.024 \mathrm{MHz}$ and shapes the noise across the Nyquist bandwidth (i.e. $0-512 \mathrm{kHz}$ ). A third-order digital low-pass Sinc filter is used to remove most of the noise at high frequency. The decimator downsamples the filtered signal to $1000 \mathrm{~Hz}$ and $250 \mathrm{~Hz}$ to be stored in a SD card and transmitted over Bluetooth, respectively. The main processing unit (MSP432) is used to (1) drive the analog front end on the sensing circuit, (2) to adjust the amplifier gain dynamically, and (3) to stream data to a host device through Bluetooth.

\section{Performance evaluation}

\subsection{BTE Signals Sensitivity Validation}

In this section, we compare the ability to capture EEG, EOG, EMG, and EDA with WAKE from BTE against the groundtruth devices from standard placements on the scalp, the eyes, the chin, and the wrist (Fig. 3). Ground-truth EEG, EOG, and EMG are measured by using an FDA-approved Lifeline Trackit Mark III device with electrodes placed at C3, C4, O1, O2, Cz, M1, M2, upper and lower parts of the left eye (VEOG), two sides of the left and right eyes (HEOG), and the chin (chinEMG), according to the International 10-20 system. Ground-truth EDA is measured by the BioPac's BioNomadix Wireless EDA Amplifier system with electrodes placed on the left wrist. The data was collected for one hour while the subject sat on a couch. We calculate Normalized Cross-Correlation (NCC) between our BTE signals with the ground-truth ones to measure the similarity between them. The measured signals are shown in Fig. 15. NCC of EEG, EOG, EMG, and EDA are as follows: Ear1-C3: 0.35, Ear1-O1: 0.28, Ear2-C4: 0.44, Ear2-O2: 0.52, Ear1-VEOG: 0.47, Ear2-HEOG: 0.59, Ear1-chinEMG: 0.62, Ear2-chinEMG: 0.76, and EarEDA-WristEDA: 0.37. The results show that Ear2, i.e. the channel crossing right and left ears, has strong correlations with scalp EEG and horizontal EOG. Ear1, i.e. the channel placed on the left ear, has a moderate correlation with scalp EEG and a strong correlation with vertical EOG. Both Ear1 and Ear2 channels have strong correlations with chin EMG. EDA on the left ear shows a moderate correlation with the signal from the wrist.

\subsection{Noise Suppression Performance}

Motion Artifacts Mitigation. We evaluated the 3CA technique in two scenarios: (1) walking and (2) sitting in a car. Each evaluation is done in one hour. Evaluation (1) consists of ten minutes of standing stationary and 50 minutes of walking in a hallway. Evaluation (2) also consists of ten minutes of sitting in a car while it is parked in a parking lot and 50 minutes of driving on an urban road (40 $\mathrm{mph}$ ). Evaluation (1) presents artifacts created by human motion while evaluation (2) presents artifacts introduced from the environment while driving. Two pairs of electrodes are put as close as possible on the same ear of a subject so that the same signals could be obtained.

Without 3CA, the BTE EOG signals (i.e. eye blinks) are completely distorted by significant motion artifacts. The noise power introduced by motion is shown in Fig. 18. During standing and parking scenarios, BTE signals with 

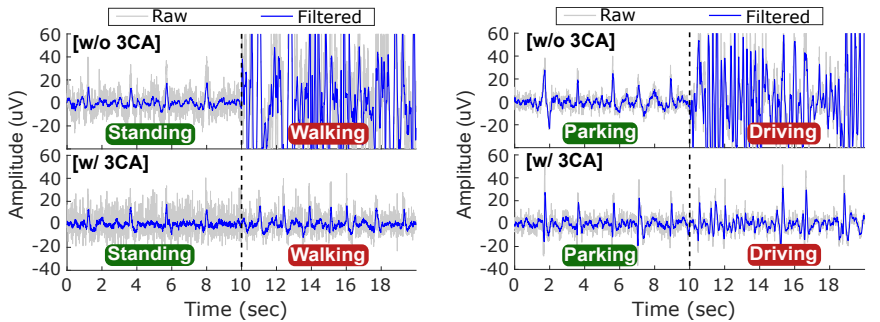

Fig. 16. Walking motion noise suppression.

Fig. 17. Driving motion noise suppression.

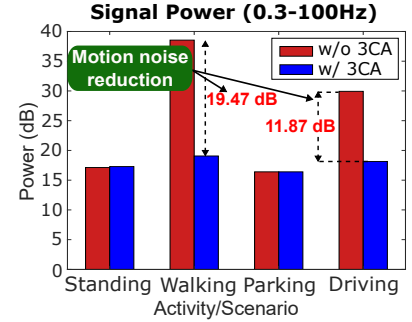

Fig. 18. Motion noise power reduction.

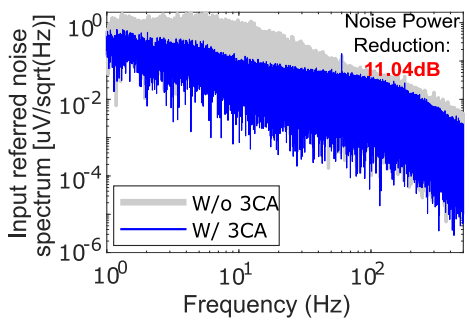

Fig. 21. 3CA noise reduction (Inside a Car).

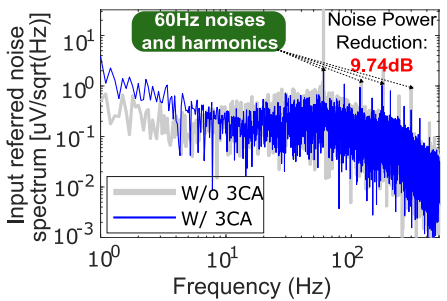

Fig. 19. 3CA noise reduction (In an Office).

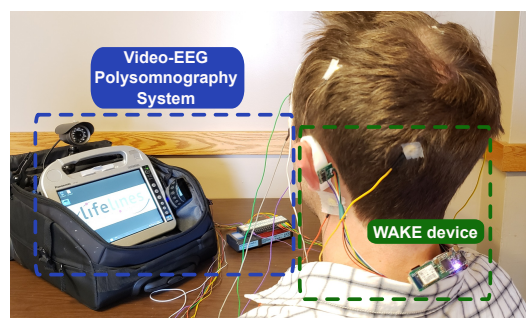

Fig. 22. Experiment setup.
Fig. 20. 3CA noise reduction (At Home)

and without 3CA have the same power. However, during walking and driving, 3CA reduces the noise power by 19.47 $\mathrm{dB}$ and $11.87 \mathrm{~dB}$. Thus, the eye blink signals are captured reliably (Fig. 16, 17).

Environmental Noise Reduction. We evaluated the ability to minimize environmental noise in three different practical environments: (1) in an office, (2) at home, and (3) inside a car. The results of the noise spectrum are shown in Fig. 19, 20, and 21, where 3CA can reduce the noise power by 9.74 to $16.1 \mathrm{~dB}$. We also found that the $60 \mathrm{~Hz}$ noise and its harmonics coupled from the electrical power line are the main sources of noise while the subject is stationary. During motion, motion artifacts are the most significant noise source at frequency ranges $0.3-100 \mathrm{~Hz}$ (Fig. 21).

\subsection{Microsleep Detection Performance}

We evaluated WAKE's ability to detect microsleep by conducting the Maintenance of Wakefulness Test (MWT), which is the existing gold-standard for quantifying microsleep [49]. We conducted three sets of experiments on the data of 19 subjects. In the first experiment, we performed the LeaveOne-Subject-Out Cross-Validation (LOSOCV), i.e. train each classifier on the set of 18 subjects and evaluate on the unseen subject. The second and third sets deal with each individual subject, in which we provided test-set and 10-folds crossvalidation. Also, we conduct these sets of experiments on 4 different epoch sizes: 3s, 5s, 7s, 9s.

TABLE 1

Demographic data of participants

\begin{tabular}{|l|l|}
\hline Age & $18-44$ years old \\
\hline Sleepiness Level & Healthy: 9, EDS: 8 , SEDS: 1, Narcolepsy: 1 \\
\hline Gender Ratio & Male: 12, Female: 7 \\
\hline
\end{tabular}

Experimental Protocol. WAKE protocol has been thoroughly designed and approved by the Institutional Review Board. 19 sleep-deprived and narcoleptic subjects on the campus were recruited for the study. Participants' demographics are shown in Tab. 1. The Sleepiness Level of each subject was recorded by using the Epworth Sleepiness Scale (ESS). The ESS score is interpreted as $<10$, healthy level; 1015, Excessive Daytime Sleepiness (EDS); and 16-24, Severe
Excessive Daytime Sleepiness (SEDS). The subjects were advised to sleep for less than five hours (only applied to subjects at the healthy level) on the night before the study and also not to consume caffeine or alcohol products before the study so that their microsleep could be faithfully captured. During each MWT session, the subject was asked to try to stay awake in a sleepiness-inducing environment. We use an FDA-Approved Video-EEG system (Lifelines Trackit Mark III) to conduct PSG as the 'ground truth'.

The MWT Protocol. We conducted two sessions of MWT for each subject with a maximum of 40 minutes each. The subject was asked to sit comfortably on a couch. The WAKE device and the 'ground-truth' PSG system were installed on them as shown in Fig. 22. We minimized all the external factors that could affect the subject's drowsiness by blocking all the light and sound coming from outside of the experiment room. The room was dark and its temperature was set at the subject's comfort levels. The subject was asked to relax but try to keep themselves awake for as long as possible, so they would not fall asleep voluntarily. The MWT starts when the light in the experiment room was turned off. We woke the subject up after they fell asleep. The collected PSG data was sent out for scoring by two certified sleep experts. To handle the variation of the manual process, one expert scored while the other expert verified independently, and the differences were resolved by discussion. Awake and microsleep episodes were marked down by following the guideline of AASM for Sleep Study [50].

Classification Evaluation Metrics. We cast the problem of microsleep detection as a binary classification problem: positive class for microsleep epoch and negative class otherwise. Here, we briefly describe four indices of the confusion matrix: true positive (TP) is the number of actual positive epochs which are correctly classified; true negative (TN) id the number of actual negative epochs which are correctly classified; false positive (FP) is the number of actual negative epochs which are incorrectly classified as positive; false negative (FN) is the number of actual positive epochs which are incorrectly classified as negative. Given these notions, we can now define precision, sensitivity, speci- 
TABLE 2

Evaluation scores with Leave-one-subject-out cross validation setting using feature-based classification, over 4 epoch sizes (3s, 5s, 7s, 9s).

\begin{tabular}{|c|c|c|c|}
\hline Epoch & Precision & Sensitivity & Specificity \\
\hline $3 \mathrm{~s}$ & 0.76 & 0.65 & 0.80 \\
\hline $5 \mathrm{~s}$ & 0.76 & 0.85 & 0.81 \\
\hline $7 \mathrm{~s}$ & 0.76 & 0.61 & 0.81 \\
\hline $9 \mathrm{~s}$ & 0.75 & 0.58 & 0.81 \\
\hline
\end{tabular}

TABLE 3

Evaluation scores with Test-Set validation setting using feature-based classification, over 4 epoch sizes (3s, 5s, 7s, 9s).

\begin{tabular}{|c|c|c|c|}
\hline Epoch & Precision & Sensitivity & Specificity \\
\hline $3 \mathrm{~s}$ & 0.83 & 0.9 & 0.81 \\
\hline $5 \mathrm{~s}$ & 0.87 & 0.9 & 0.87 \\
\hline $7 \mathrm{~s}$ & 0.88 & 0.93 & 0.86 \\
\hline 9s & 0.89 & 0.95 & 0.88 \\
\hline
\end{tabular}

ficity scores as follow: Precision $=\frac{T P}{T P+F P} ;$ Sensitivity $=$ $\frac{T P}{T P+F N} ;$ Specificity $=\frac{T N}{T N+F P}$. Due to the nature of our detection problem (the number of microsleep epochs is much less than that of awake ones), precision, sensitivity, and specificity are preferred over the accuracy index.

Data summary. Our dataset contains 19 subjects. We then segment and label each epoch based on the epoch size. For instance, with $5 \mathrm{~s}$ epoch ( $80 \%$ overlap), our data consists of 35,558 and 8,845 samples for awake and microsleep states, respectively. The ratio of negative:positive is approximately $4: 1$, as an essence of rare microsleep events. This imbalance problem is known to severely affect the performance of popular classification algorithms. Thus, we downsampled the awake set to the same amount of microsleep data in each experiment and put this imbalance ratio (number of negative epochs/number of positive epochs) in the weighted cost during training. For example, in the first iteration of the LOSOCV experiment, we left subject 18 out for testing and we pooled samples of all training subjects, which consists of 32,778 negative samples and 8,572 positive samples. We downsampled the negative samples to 8,572 instances (same as the positive one) and used the weighted cost of $32,778: 8,572$ for training. For feature-based classification, we perform experiments on 4 epoch sizes: $3 \mathrm{~s}, 5 \mathrm{~s}, 7 \mathrm{~s}$, $9 \mathrm{~s}$. With the recommended epoch size of $5 \mathrm{~s}$, we illustrate the promising results of deep neural networks on learning micro-sleep from the collected signals.

Set 1: Leave-one-subject-out cross validation: we alternatively trained our classifiers on the data pool of 18 subjects and evaluated the trained model on the remaining subject. The final scores are the average over these 19 iterations. Table 2 and Table 5 (row 1) present our results on this setting for feature-based learning and deep learning respectively. The hierarchical classifier achieved the best performance among examined classifiers, obtaining approximately 0.76 on precision and just over 0.8 on specificity for all epoch sizes. The model has a slight variation on sensitivity scores in which the recommended size of 5-second results in the highest sensitivity of 0.85 . This result is expected as large value shifts are known to happen across different subjects. Nevertheless, this result shows the feasibility of WAKE for microsleep detection on unseen subjects.

Set 2: Test-set on each subject (Test-set): we applied stratified split onto data of each subject, dividing them into two parts with the ratio $75 \%$ (training): $25 \%$ (testing) with respect to the percentage of positive and negative samples. We then trained our classifiers on the training
TABLE 4

Evaluation scores with 10 -fold cross validation setting using feature-based classification, over 4 epoch sizes (3s, 5s, 7s, 9s).

\begin{tabular}{|c|c|c|c|}
\hline Epoch & Precision & Sensitivity & Specificity \\
\hline $3 \mathrm{~s}$ & 0.84 & 0.89 & 0.83 \\
\hline $5 \mathrm{~s}$ & 0.88 & 0.89 & 0.96 \\
\hline $7 \mathrm{~s}$ & 0.88 & 0.93 & 0.87 \\
\hline 9s & 0.9 & 0.94 & 0.90 \\
\hline
\end{tabular}

TABLE 5

Evaluation scores using Deep neural network on raw data with epoch size $=5 \mathrm{~s}$. We evaluated on 3 test settings.

\begin{tabular}{|c|c|c|c|}
\hline Setting & Precision & Sensitivity & Specificity \\
\hline LOSOCV & 0.56 & 0.45 & 0.65 \\
\hline Test-Set & 0.86 & 0.85 & 0.86 \\
\hline 10-Folds CV & 0.88 & 0.89 & 0.88 \\
\hline
\end{tabular}

data and evaluated the performance on the test set. Table 3 and Table 5 (row 2) present our results on this setting for feature-based learning and deep learning respectively. Among simple classifiers, RandomForest models with 20 estimators constantly achieved the best scores for each of the epoch size. Compared to Setting 1 (LOSOCV), this setting results in better evaluated scores (above 0.8 for precision and nearly 0.9 for sensitivity), which can explained by the high similarity between data within a certain subject.

Set 3: 10-fold cross validation: we conducted crossvalidation for each subject's data and averaged the scores for the final results. Specifically, for cross-validation on a particular subject, we left $1 / 10$ of the data for evaluation and trained on the remaining data. This procedure was performed 10 times before we got the average scores as the representative. Table 4 and Table 5 (row 3) show our results on this setting for feature-based learning and deep learning respectively. Similar to Test-set setting, our Random Forest classifiers were able to achieve high scores on precision, sensitivity, and specificity over all of the subjects.

Compared to feature-based classification, deep learning models show promising and comparable performance, especially on test-set and k-fold classification. Fig. 23 presents the learning curves of training SorNet on the test-set setting. After 200 epochs, the training loss and precision curves reach a point of stability. The training loss, training precision, and validation precision converge to $0.015,0.998$, and 0.897 , respectively. Our learning curves show that the network is sufficiently expressive and capable of learning the target classification function over the data. Poor performance of our deep networks on on the leave-one-subject-out setting is expected due to the highly complex pattern of brain signals and the challenging cross-subject phenomenon. Regarding the size of epoch, 5-second constantly leads to reliable performance: high precision and high sensitivity with respect to the average scores, though increasing epoch size may improve performance in some test settings.

Performance on Mobile Devices: To evaluate the performance of our developed Machine Learning algorithm on a mobile device, we deploy both the features-based and deep neural network (DNN) models on a Samsung Galaxy S10 (Android 11, 1.95-2.73GHz Octa-core CPU, 128GB Flash, 8GB RAM). For each model, we run the classification 100 times and measure the maximum memory usage and inference latency. Table 6 presents our experimental results with the maximum memory usage and latency are $118 \mathrm{MB}$ and $72 \mathrm{~ms}$, respectively. The latency of the feature-based model is a bit higher than the DNN model since we could not find 

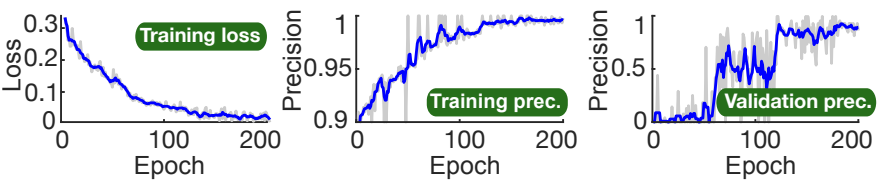

Fig. 23. Learning curves of training SorNet on the test-set setting.

equivalent native Android libraries. Thus, it is deployed inside a virtual Linux environment on Android. Nevertheless, we can see from the results that the computational overhead of both feature-based and deep learning models is not significant and the developed models can be readily deployed on mobile platforms.

\subsection{Usability Analysis}

WAKE Prototype Power Usage. We measured the power consumption of the WAKE prototype by using a Monsoon Power Monitor device with the sampling rate at $5 \mathrm{kHz}$. Each measurement was done in $180 \mathrm{~s}$, resulting in $900 \mathrm{k}$ data points, to get a stable result. At $25^{\circ} \mathrm{C}$ and $3.7 \mathrm{~V}$ nominal battery voltage, the average power usage of WAKE is as follows: (1) Active state (real-time biosignals streaming with Bluetooth) consumes $241.5 \mathrm{~mW}$, and (2) Idle state (no streaming with only MCU is kept running in idle mode while other components are turned off) consumes $51.60 \mathrm{~mW}$. With a $600 \mathrm{mAh}$ Li-Po battery, WAKE prototype can operate for 9.2 hours in Active, and stay in Idle for 43.1 hours. Further component-level measurements of usage power during Active were done by turning off each component one by one and repeating the measurements. Fig. 24 presents a full active power usage breakdown of the WAKE device. The sensing components (amplifiers and external ADCs) and Bluetooth communication module consume the most of system power with the average of $93.5 \mathrm{~mW}$ and $85.2 \mathrm{~mW}$, respectively. The storage module (uSD card) will increase an additional $90.2 \mathrm{~mW}$ if it is turned on. The processing unit only consumes $62.8 \mathrm{~mW}$. These numbers show the capability of our WAKE prototype to monitor the user's microsleep during a long duration. They could be further lowered by optimizing Bluetooth transmission parameters and taking advantage of deep power saving modes of the MCU.

WAKE Prototype Thermal Profiling. We conducted thermal measurement for the processing unit of our WAKE prototype for 14 hours continuously. The measurement was designed to emulate the scenario where continuous microsleep monitoring is needed during normal working hours. It was divided into three states: (1) idle (the device waits for a Bluetooth connection), (2) streaming (the device streams the measured biosignals to both its onboard uSD Card and a Bluetooth-connected mobile device), (3) standby (the device stops data streaming but its Bluetooth connection is still available for future commands). The idle, streaming, and standby states lasted for 1, 12, 1 hour, respectively. Thermal data was measured by the internal temperature sensor of the processing unit and reported every 5s. Fig. 25 presents our measurement results. On average, the temperature of idle and standby states are 31.65 and 35.75 degrees Celsius, respectively. During streaming, the temperature increases to an average of 37.38 degrees and the peak is 38.9 degrees. According to the standard of American Society for Testing and Materials, 43 degrees Celsius is the threshold for prolonged use (i.e. $>8 h$ ) on human skin without creating
TABLE 6

Classification performance on a Galaxy S10.

\begin{tabular}{|l|l|l|}
\hline & Memory Usage & Latency (avg./std.) \\
\hline Feature-based & $74 \mathrm{MB}$ & $72.4 / 19.5 \mathrm{~ms}$ \\
\hline DNN & $118 \mathrm{MB}$ & $5.74 / 1.93 \mathrm{~ms}$ \\
\hline
\end{tabular}

any injury [51]. The temperature of our WAKE prototype is always below this threshold.

User Study. We conducted a survey to evaluate WAKE's usability. We distributed our survey to the 19 subjects in our MWT study and 17 other people on the campus after they have used the WAKE device for at least two hours. In total, 36 people answered our survey. Fig. 26a presents the questions that we used to ask our participants' opinions on their experience with the WAKE device. The results show that over $85 \%$ of people felt comfortable with our WAKE device and were willing to wear it during daily mental fatigue tasks, such as during driving, night-time working, etc. $91.6 \%$ of people agree that the WAKE device is more comfortable than the 'ground-truth' device used in PSG. $62.5 \%$ of people found it easy to use the WAKE device, while $16.7 \%$ had some difficulties with skin preparation and putting on the conductive paste.

We noticed that people with eyeglasses are most likely to be affected by wearing WAKE, as both devices need to be rested on users' ears. Thus, to evaluate the compatibility of WAKE and eyeglasses during daily activities, an additional users' study on a population of eight people was conducted. In this study, we asked the users to wear both WAKE and eyeglasses during their daily working time for 3-4 hours. They were asked to wear WAKE before wearing eyeglasses so that the eyeglasses' temple tips could sit on top of the WAKE's silicone earpieces. The survey questions and results are presented in Fig. 26b. All users reported that they did not feel disturbed during their normal activity, and they can easily wear both WAKE and eyeglasses. $75 \%$ of users agree that it was comfortable to wear both devices for long hours thanks to the softness of the silicon. Only two users had slight discomfort because of additional weight and the gripping force of WAKE earpieces.

\section{Related WORKS}

Existing microsleep detection systems mainly use scalp EEG, eye tracking with EOG or cameras, IMU, and infrared light. EEG and EOG signals have been widely used to detect microsleep [52, 53]. However, the conventional devices used to captured those signals can only be used in a controlled environment and are not socially acceptable. Camera-based approaches detect microsleep by analyzing the movement of head and eyes [9], or pupils' dilation [54]. IMU sensors can be used to approximate body motion corresponding to microsleep (smartwatch [55], hairband [10]). In addition, infrared light reflection methods monitor the eyelid movement of the subject such as Vigo [56], BlinQ [57]. These devices cannot recognize the inner physiological state and its reliability has not been thoroughly evaluated.

In literature, there are also many drowsiness detection and monitoring works such as $[58,59,60]$. Drowsiness and microsleep detection works, however, should not be treated equally. In particular, drowsiness is a physiological state that is defined when there exists a sleep pressure, which may cause slower reaction time or compromise vision but 


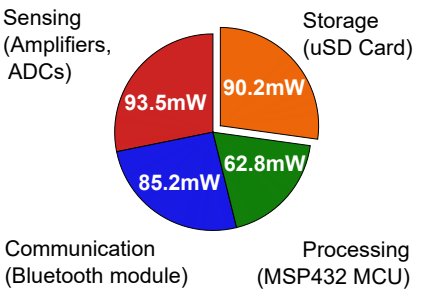

Fig. 24. Active power usage.

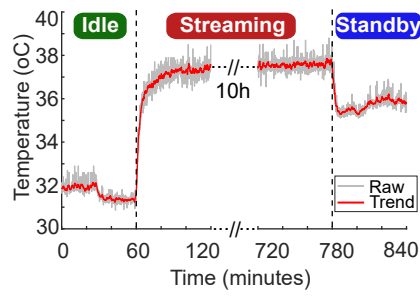

Fig. 25. CPU core thermal profile.

does not mean fatal as our brain is still conscious of the surrounding environment [11]. Microsleep, on the other hand, is the brief and often fatal duration, where the brain loses consciousness [20]. Additionally, drowsiness detection works (especially in driving scenarios) use various methods to quantify the existence of drowsiness such as steering pattern monitoring, vehicle position in lane monitoring, driver eye/face monitoring, etc. [58], which are different from ones (i.e. brain activity represents microsleep period) used for microsleep. Particularly, in [59], the authors detect drowsiness by quantifying wake/sleep epochs, however, they also state that their system is not sensitive enough to detect microsleep events. In [60], lane deviation in a driving simulation, which is pointed out in [58] that it is not a reliable metric, is used as the indicator of drowsiness.

cEEGrids [23] and TYTH [25] demonstrated the feasibility to capture EEG/EMG from behind the ear. However, the ability to detect microsleep from BTE sensors has not been evaluated before. Vital signs monitoring using wearable and mobile sensors has also been investigated in various studies such as breathing measurement [61], stress estimation (heart rate variability, galvanic skin response, and EMG) [26], and eating detection (IMU, microphone, and proximity) $[62,63]$. However, to the best of our knowledge, there are no existing works to detect microsleep from wearable BTE sensors accurately and reliably.

\section{Discussions}

In-the-wild evaluation. With the promising results from our in-lab evaluation, we aim for a larger scale out-lab evaluation. One of the key challenges is the limitation of the existing ground-truth for microsleep detection. Up to now, the gold standard to objectively assess microsleep is based on polysomnographic (PSG) data, which can only be conducted in a controlled environment. Ground-truths based on pupil dilation or eye-tracking have potentials and are directions worth exploring.

Impact of sweat condition. While WAKE can address motion and environmental noises, there are several artifacts posing as challenges to the real-world usability of a wearable system like WAKE. For instance, sweating and hydration can introduce noises into the measurement. Addressing these artifacts is the question that we will explore to enhance the practicality of WAKE.

Optimizing WAKE device. The current prototype is designed with off-the-shelf materials and components. Hence, it is challenging to ensure the manufacturing quality of our customized earpieces, electrodes, and the sensing circuit. The use of wet electrodes is also not desirable during daily usages because of the additional steps needed to apply the conductive paste. Besides, the current power consumption is still high. Thus, improving the quality of our cus-

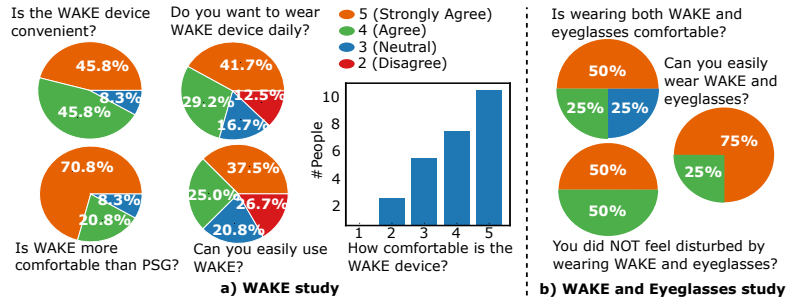

Fig. 26. User study.

tomized components, optimizing power consumption, employing dry electrodes, and a better mechanism to maintain electrode-skin contact are important tasks to further increase the usability of the system.

Trade-off between classification performance and latency. Though highly complex prediction models may improve the accuracy, they usually take larger amount of computations because of the complex processes. We, thereby, make use of simpler off-the-shell classifiers which are deployed and optimized commonly in latency-sensitive libraries. Another key part of our system is to use highly informative expert-based features which are simple and efficient to compute. These settings help us reduce the processing and inference time, shortening the latency toward warning the user.

Feasibility of deep learning on raw data. Deep neural networks (DNNs) are designed to automatically extract features from raw bio-signal data. This is a huge advantage compared to other classical machine learning algorithms, which require labor-intensive domain-crafted features. Shown in Table 5, DNNs achieve high detection accuracies for both test-set and 10-fold CV evaluations, which are as good as the performance of feature-based models (Tables 4, 3). However, we observe a non-trivial drop of performance in LOSOCV setting. Notice that the LOSOCV is highly challenging because bio-signal data come from different subjects. We hypothesize that this drop is due to large variance of inter-subjects' signals, outliers in physiological signals, and the lack of training data for DNNs. On the other hand, classical algorithms utilize embedded prior knowledge in hand-designed features to generalize better, in the low-data regime. Nevertheless, our results illustrate the promise of DNNs in detecting micro-sleep using our WAKE data, and leaves an open question on improving its generalization over different subjects.

\section{CONCLUSION}

We presented WAKE, a novel compact, lightweight, and socially acceptable wearable device to detect microsleep from behind the ears. We proposed the Three-fold Cascaded Amplifying technique to remove the impact of motion artifacts and environmental noises. We evaluated the motion and environmental noise suppression and microsleep detection performance on 19 subjects. WAKE can reduce noise power by $9.74-19.47 \mathrm{~dB}$ in different practical scenarios such as walking, driving. We develop a classification model based on the core biomarkers of microsleep captured by WAKE. WAKE achieves $76 \%$ precision and $85 \%$ recall in detecting microsleep in LOSOCV.

\section{ACKNOWLEDGMENTS}

We want to thank the editors and anonymous reviewers for the insightful feedback on our manuscript. We also would 
like to thank Ms. Carole Kline, Dr. Scott Holman, Vachan D A, Frederick Thayer, Evan Stene, Anh Nguyen, and Katrina Siegfried for your valuable supports with the project. This research was partially funded by NSF CNS/CSR \#1846541, NSF SCH \#1602428, and Amazon \#2012BL005/JL7. A preliminary version of this article appears in the Proceedings of The 18th ACM International Conference on Mobile Systems, Applications and Services.

\section{REFERENCES}

[1] J.F. Pagel. Excessive Daytime Sleepiness, May 2009. https://www. aafp.org/afp/2009/0301/p391.html.

[2] Katrin Uehli, Amar J Mehta, et al. Sleep problems and work injuries: a systematic review and meta-analysis. Sleep medicine reviews, 18(1):61-73, 2014.

[3] Drew Dawson, Amy C Reynolds, et al. Determining the likelihood that fatigue was present in a road accident: a theoretical review and suggested accident taxonomy. Sleep medicine reviews, 2018.

[4] American Academy of Sleep Medicine. Economic burden of undiagnosed sleep apnea in U.S. is nearly $\$ 150 \mathrm{~B}$ per year. https: //tinyurl.com/yxkfe5zh.

[5] Poul Jennum, Stine Knudsen, et al. The economic consequences of narcolepsy. Journal of Clinical Sleep Medicine, 5(03):240-245, 2009.

[6] American Addiction Centers. Narcolepsy and How Substance Abuse Effects It, 2018. https://tinyurl.com/yxh4hh7z.

[7] Marco Hafner, Martin Stepanek, Jirka Taylor, et al. Why sleep matters - the economic costs of insufficient sleep: a cross-country comparative analysis. Rand health quarterly, 6(4), 2017.

[8] U.S. Department of Health and Human Services. Types of Sleep Studies. https://tinyurl.com/y232zaac.

[9] Zutao Zhang and Jiashu Zhang. A new real-time eye tracking based on nonlinear unscented kalman filter for monitoring driver fatigue. Journal of Control Theory and Applications, 8(2):181-188, 2010.

[10] Esra Vural, Mujdat Cetin, Aytul Ercil, et al. Drowsy driver detection through facial movement analysis. In International Workshop on Human-Computer Interaction, pages 6-18. Springer, 2007.

[11] Carskadon Roehrs et al. Daytime sleepiness and alertness. In Principles and Practice of Sleep Medicine: Fifth Edition. Elsevier Inc., 2010.

[12] Christian Guilleminault, Michel Billiard, Jacques Montplaisir, et al. Altered states of consciousness in disorders of daytime sleepiness. Journal of the neurological sciences, 26(3):377-393, 1975.

[13] Vivian Genaro Motti and Kelly Caine. Users' privacy concerns about wearables. In International Conference on Financial Cryptography and Data Security, pages 231-244. Springer, 2015.

[14] The Guardian. Google Glass security failings may threaten owner's privacy. https://tinyurl.com/y4xopov9.

[15] Investopedia. How and Why Google Glass Failed. https://tinyurl. $\mathrm{com} / \mathrm{y} 3 \mathrm{bqxtlm}$

[16] Kazumi Takahashi, J-S Lin, and Kazuya Sakai. Neuronal activity of orexin and non-orexin waking-active neurons during wake-sleep states in the mouse. Neuroscience, 153(3):860-870, 2008.

[17] Takeshi Sakurai. The neural circuit of orexin (hypocretin): maintaining sleep and wakefulness. Nature Reviews Neuroscience, 8(3):171, 2007.

[18] Malik TR Peiris et al. Frequent lapses of responsiveness during an extended visuomotor tracking task in non-sleep-deprived subjects. Journal of sleep research, 15(3):291-300, 2006.

[19] Amit Paul et al. Variability of driving performance during microsleeps. 2005.

[20] Laura Higgins and Bernie Fette. Drowsy driving. 2012.

[21] The PSG Reading Center. Procedure manual for polysomnography. https://tinyurl.com/yc9ptdjz.

[22] Andrew D Nordin et al. Dual-electrode motion artifact cancellation for mobile electroencephalography. Journal of neural engineering, 2018.

[23] Martin G Bleichner and Stefan Debener. Concealed, unobtrusive earcentered eeg acquisition: ceegrids for transparent eeg. Frontiers in human neuroscience, 11:163, 2017.

[24] Antoine Favre-Félix et al. Absolute eye gaze estimation with biosensors in hearing aids. Frontiers in neuroscience, 13:1294, 2019.

[25] Phuc Nguyen, Nam Bui, et al. Tyth-typing on your teeth: Tongueteeth localization for human-computer interface. In Proceedings of the 16th Annual International Conference on Mobile Systems, Applications, and Services, pages 269-282. ACM, 2018.

[26] Wen Wen, Daisuke Tomoi, Hiroshi Yamakawa, Shunsuke Hamasaki, Kaoru Takakusaki, Qi An, Yusuke Tamura, Atsushi Yamashita, and Hajime Asama. Continuous estimation of stress using physiological signals during a car race. Psychology, 8(07):978, 2017.
[27] Caroline J Smith and George Havenith. Body mapping of sweating patterns in male athletes in mild exercise-induced hyperthermia. European journal of applied physiology, 111(7):1391-1404, 2011.

[28] Bernard Grundlehner et al. Ambulatory eeg monitoring. 2019.

[29] Jiawei Xu, Srinjoy Mitra, Chris Van Hoof, et al. Active electrodes for wearable eeg acquisition: Review and electronics design methodology. IEEE reviews in biomedical engineering, 10:187-198, 2017.

[30] Evangelia-Regkina Symeonidou et al. Effects of cable sway, electrode surface area, and electrode mass on electroencephalography signal quality during motion. Sensors, 18(4):1073, 2018.

[31] AB Simakov et al. Motion artifact from electrodes and cables. 2010.

[32] AC Metting Van Rijn et al. High-quality recording of bioelectric events. Medical and Biological Engineering and Computing, 28(5), 1990.

[33] Emily S Kappenman and Steven J Luck. The effects of electrode impedance on data quality and statistical significance in erp recordings. Psychophysiology, 47(5):888-904, 2010

[34] Esrafil Jedari, Rashid Rashidzadeh, Mitra Mirhassani, et al. Twoelectrode ecg measurement circuit using a feed forward cmrr enhancement method. In 2017 IEEE 30th Canadian Conference on Electrical and Computer Engineering (CCECE), pages 1-4. IEEE, 2017.

[35] R. Juszkiewicz. Achieving a Fully Differential Output Using SingleEnded Instrumentation Amplifiers. https://tinyurl.com/y4klzq6h.

[36] James C Huhta and John G Webster. 60-hz interference in electrocardiography. IEEE Transactions on Biomedical Engineering, 1973.

[37] Richard G Lyons. Understanding digital signal processing, 3/E Pearson Education India, 2004.

[38] Carlo I De Luca. Surface electromyography: Detection and recording. DelSys Incorporated, 10(2):1-10, 2002.

[39] Muhammad Zahak Jamal. Signal acquisition using surface emg and circuit design considerations for robotic prosthesis. Computational Intelligence in Electromyography Analysis-A Perspective on Current Applications and Future Challenges, 18:427-448, 2012

[40] Joshua D Reiss. Understanding sigma-delta modulation: the solved and unsolved issues. Journal of the Audio Engineering Society, 56(1/2):49-64, 2008.

[41] Mathias Benedek and Christian Kaernbach. Decomposition of skin conductance data by means of nonnegative deconvolution. Psychophysiology, 47(4):647-658, 2010.

[42] S. Ozs̃en et al. Examining the effect of time and frequency domain features of eeg, eog, and chin emg signals on sleep staging. In 201015 th National Biomedical Engineering Meeting, pages 1-4, April 2010.

[43] Lan lan Chen, Yu Zhao, Jian Zhang, and Jun zhong Zou. Automatic detection of alertness/drowsiness from physiological signals using waveletbased nonlinear features and machine learning. Expert Systems with Applications, 42(21):7344 - 7355, 2015.

[44] B. Sen et al. A Comparative Study on Classification of Sleep Stage Based on EEG Signals Using Feature Selection and Classification Algorithms. Journal of Medical Systems, 38(3):1-21, 2014.

[45] Arnaud Sors, Stéphane Bonnet, et al. A convolutional neural network for sleep stage scoring from raw single-channel eeg. Biomedical Signal Processing and Control, 42:107-114, 2018.

[46] Kevin P Murphy. Machine learning: a probabilistic perspective. MIT press, 2012.

[47] Diederik P Kingma and Jimmy Ba. Adam: A method for stochastic optimization. arXiv preprint arXiv:1412.6980, 2014.

[48] Patrick K Sullivan, Michael J Brucker, and Jagruti Patel. A morphometric study of the external ear: Age and sex related differences, 2010.

[49] Bernie Y Sunwoo, Nicholas Jackson, Greg Maislin, Indira Gurubhagavatula, Charles F George, and Allan I Pack. Reliability of a single objective measure in assessing sleepiness. Sleep, 35(1):149-158, 2012.

[50] Conrad lber et al. The AASM manual for the scoring of sleep and associated events: rules, terminology and technical specifications. American Academy of Sleep Medicine Westchester, IL, 2007.

[51] A. Han. Thermal management and safety regulation of smart watches. In 2016 15th IEEE Intersociety Conference on Thermal and Thermomechanical Phenomena in Electronic Systems. IEEE, 2016.

[52] Martin Golz, Adolf Schenka, Florian Haselbeck, et al. Inter-individual variability of eeg features during microsleep events. Current Directions in Biomedical Engineering, 5(1):13-16, 2019.

[53] Abdul Baseer Buriro, Reza Shoorangiz, et al. Predicting microsleep states using eeg inter-channel relationships. IEEE Transactions on Neural Systems and Rehabilitation Engineering, 2018.

[54] Luis Miguel Bergasa, Jesús Nuevo, Miguel A Sotelo, Rafael Barea, et al. Real-time system for monitoring driver vigilance. IEEE Transactions on Intelligent Transportation Systems, 7(1):63-77, 2006.

[55] Boon-Leng Lee et al. Standalone wearable driver drowsiness detection system in a smartwatch. IEEE Sensors journal, 16(13), 2016. 
[56] Vigo. Vigo smart headset. https://tinyurl.com/y3jodh6u.

[57] BlinQ. Blinq system. https://tinyurl.com/yxlkcsr5.

[58] Arun Sahayadhas et al. Detecting driver drowsiness based on sensors: a review. Sensors, 12(12):16937-16953, 2012.

[59] Walter Karlen. Adaptive wake and sleep detection for wearable systems. Technical report, EPFL, 2009.

[60] Chin-Teng Lin, Che-Jui Chang, et al. A real-time wireless braincomputer interface system for drowsiness detection. IEEE transactions on biomedical circuits and systems, 4(4):214-222, 2010.

[61] Rajalakshmi Nandakumar, Shyamnath Gollakota, and Nathaniel Watson. Contactless sleep apnea detection on smartphones. In Proceedings of the 13th annual international conference on mobile systems, applications, and services, pages 45-57. ACM, 2015.

[62] Abdelkareem Bedri, Richard Li, et al. Earbit: using wearable sensors to detect eating episodes in unconstrained environments. Proceedings of the ACM on interactive, mobile, wearable and ubiquitous technologies, 1(3):37, 2017.

[63] Shengjie Bi, Tao Wang, et al. Auracle: Detecting eating episodes with an ear-mounted sensor. Proceedings of the ACM on Interactive, Mobile, Wearable and Ubiquitous Technologies, 2(3):1-27, 2018.

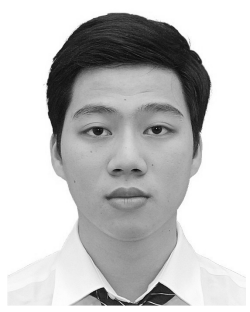

Nhat Pham is a DPhil student at the Department of Computer Science, University of Oxford. He received his $\mathrm{BE}$ in Computer Engineering from VNUHCM - University of Technology in 2015 and his MS in Computer Science from KAIST, South Korea in 2018. His research interests include cyber-physical systems, mobile health sensing, operating systems, cognitive radio networks, and wireless sensor networks.

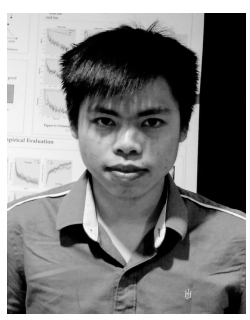

Tuan Dinh is a PhD student at the Department of Computer Science, University of Wisconsin Madison. He received his BE in Computer Science from VNUHCM - University of Technology in 2015. His research interests include machine learning, representation learning, deep generative models and applied research in biomedical science.

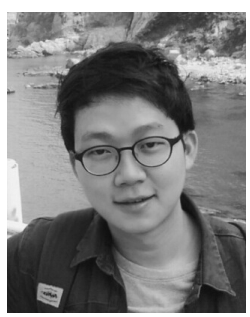

Taeho Kim is a PhD student at the Department of Computer Science, University of Colorado, Boulder. He received his BS from Korea University, South Korea in 2018. His research interests include mobile healthcare, Internet of Things system, and mobile computing.

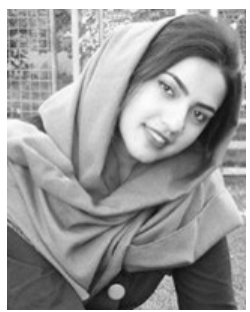

Zohreh Raghebi has been working toward her $\mathrm{PhD}$ degree at the Department of Computer Science and Engineering, University of Colorado Denver since 2015. She received her MS degree in Computer Science from the University of Tehran in 2014. Her current research interest includes big data mining and management on large scale graphs, and machine learning with an emphasis on biomedical and bioinformatics applications.

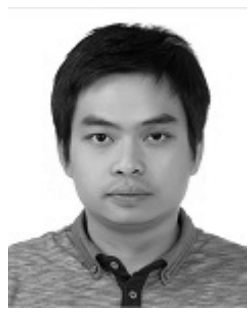

Nam Bui is a PhD student at the Department of Computer Science, University of Colorado, Boulder. He received his MS at The Nottingham University in Computer Science in 2012 and a BS at VNUHCM - University of Science in Mathematics in 2012. His research interests include computational health, light- sensing systems, human physiological signal sensing, and computer vision.

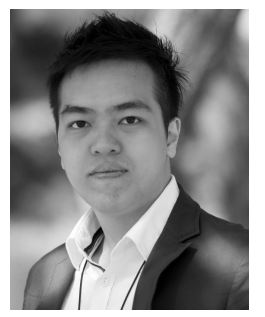

Hoang Truong received his BS in Electrical \& Electronic and MS in Computer Science from KAIST, South Korea, in 2012 and 2014 respectively. $\mathrm{He}$ is pursuing a $\mathrm{PhD}$ in Computer Science at the University of Colorado Boulder. His research interests include mobile healthcare, wearable sensing and wireless communication.

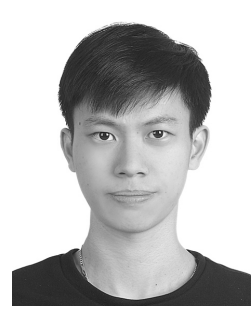

Tuan Nguyen is a DPhil student at the Department of Computer Science, University of Oxford. He received his BS from UNIST, South Korea in 2019 and his MS from KAIST, South Korea in 2020. His research interests include stochastic deep learning, deep learning for mobile devices, and mobile healthcare.

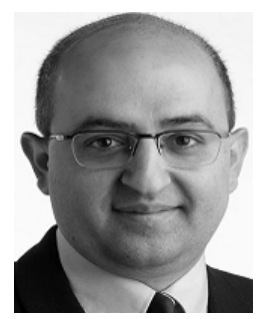

Farnoush Banaei-Kashani is an Assistant Professor at the Department of Computer Science and Engineering, University of Colorado Denver. He earned his PhD degree in Computer Science and MS degree in Electrical Engineering at the University of Southern California in 2007 and 2002 , respectively. He is passionate about performing fundamental research toward building practical, large-scale, data-intensive systems, with particular interest in Data-driven Decisionmaking Systems.

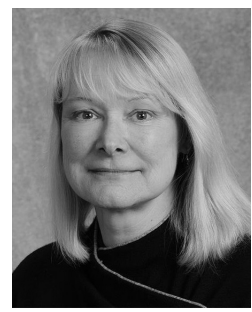

Ann Halbower is a pediatric pulmonologist in Aurora, Colorado, and is affiliated with multiple hospitals in the area, including Children's Hospital Colorado and University of Colorado Hospital. She received her medical degree from University of Massachusetts Medical School and has been in practice for more than 20 years.

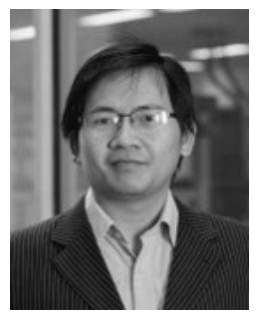

Thang Dinh received his BS in IT from Vietnam National University, Vietnam in 2007, and PhD in Computer Engineering from the University of Florida in 2013. He is currently an Associate Professor at the Department of Computer Science, Virginia Commonwealth University. His research interests are block- chain optimization, social networks and billion-scale graph mining, network security and resiliency, and approximation algorithm.

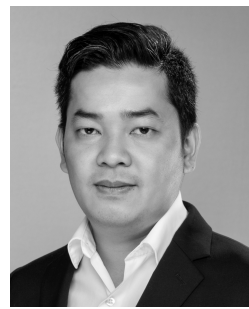

Phuc Nguyen obtained his BS in Electronics \& Telecommunications from VNUHCM-University of Science in 2010, his MS in Electrical \& Computer Engineering from Sungkyunkwan University, S. Korea in 2014, and his PhD in Computer Science from University of Colorado Boulder in 2018. He is currently an Assistant Professor at the Department of Computer Science and Engineering, University of Texas Arlington. His research interests include cyber-physical systems, mobile healthcare, and wireless technologies.

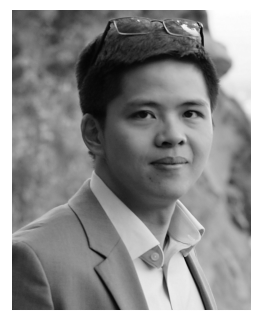

Tam Vu received his BS in Computer Science from Hanoi University of Technology, Vietnam in 2006, and his $\mathrm{PhD}$ in Computer Science from WINLAB, Rutgers University in 2013. He is currently an Associate Professor at the Department of Computer Science, University of Oxford and University of Colorado Boulder, and the Director of Mobile and Networked Systems Lab. His research interests include wireless sensing, mobile healthcare, mobile centric network, mobile communication, and mobile security. 